\title{
Construction and Borel Summability of Planar 4-Dimensional Euclidean Field Theory
}

\author{
Vincent Rivasseau \\ Centre de Physique Théorique de 1'Ecole Polytechnique, Plateau de Palaiseau, \\ F-91128 Palaiseau, Cedex, France
}

\begin{abstract}
We use the methods of [1] to show that the planar part of the renormalized perturbation theory for $\varphi_{4}^{4}$-euclidean field theory is Borelsummable on the asymptotically free side of the theory. The Borel sum can therefore be taken as a rigorous definition of the $N \rightarrow \infty$ limit of a massive $N \times N$ matrix model with a $+\operatorname{tr} g \varphi^{4}$ interaction, hence with "wrong sign" of $g$. Our construction is relevant for a solution of the ultra-violet problem for planar QCD. We also propose a program for studying the structure of the "renormalons" singularities within the planar world.
\end{abstract}

\section{Introduction}

The standard problem in constructive field theory is to prove the existence of "models" which represent realistic interacting fields. Ultimately it should give a rigorous mathematical construction of the models which are used in the description of modern particle physics, namely the gauge theories. So far constructive field theory has not been able to provide the construction of any interacting model in 4 dimensions of space-time. To construct the 4-dimensional theories requires in our opinion a complete analysis of their relationship to renormalized perturbation theory. This does not mean that we do not believe in the existence of "non perturbative" effects in 4-dimensional gauge theories. There is good heuristic evidence for the existence of such effects. However we think that it is unlikely that a rigorous construction of these theories in the continuum can be obtained before the perturbative phenomena have been investigated in detail and brought under rigorous control.

It is known in particular that in 4-dimensional renormalizable field theories the renormalization deeply modifies the behavior of Feynman amplitudes. Some individual amplitudes become so large that alone they seem to dominate the large order behavior of perturbation theory. We think that this phenomenon, which has been analyzed in various heuristic ways [2-6], should be rigorously understood and controlled. A first step in this direction was accomplished in [1], where the 
necessary methods were developed to evaluate systematically and rigorously the size of the renormalization effects. To go further one would like to have rigorous information on the existence, the position and the analytic structure of the singularities that these large renormalization effects may induce, and to which we give the generic name of "renormalons" after [4]. We regard the present paper as a step in this direction. Using the methods of [1] we provide a rigorous perturbative construction of planar 4-dimensional massive asymptotically free field theories at weak coupling by proving the Borel summability of their perturbative expansion ${ }^{1}$. Since it is very likely that the renormalon singularities are responsible for the divergence of this expansion, the fact that we are able to prove its Borel summability is an indication that these singularities might be controlled by perturbative methods. In fact we propose to see the planar world as a laboratory to study in detail the heuristic results about both infra-red and ultra-violet renormalons. We explain this proposal in more detail in Sect. IV.

Before we describe further our results let us emphasize what makes them truly different from other results on Borel summability of perturbative expansions in quantum mechanics or quantum field theory [12-16]. Let us consider the standard Euclidean scalar field with a $+g \varphi^{4}$ interaction in dimension $d$ of space time. In the rest of this paper we adopt the unusual but useful convention that this $+g \varphi^{4}$ interaction is in the Lagrangian, hence it corresponds to the "wrong" negative sign of $\varphi^{4}$ in the Hamiltonian. The renormalized perturbation expansion in the coupling constant for a Schwinger function $S$ can be written as:

$$
\begin{gathered}
S=\sum_{n=1}^{\infty} g^{n} a_{n}, \\
a_{n}=\sum_{G / n(G)=n} I_{G}^{R},
\end{gathered}
$$

which means that the $n^{\text {th }}$ order of the perturbative expansion, $a_{n}$, is the sum over all graphs with $n$ vertices of the corresponding renormalized Feynman amplitudes $I_{G}^{R}$. However, one cannot take directly (I.1) as a rigorous definition of $S$ because the radius of convergence in $g$ of the right-hand side of (I.1) should be 0 [17] [proofs of this statement have been obtained only for superrenormalizable interactions: $P(\varphi)_{2}$ [18] and $\varphi_{3}^{4}$ [19]]. Nevertheless in the superrenormalizable case a connection does exist between the two members of Eq. (I.1); it has been proved that the left-hand side is quite generally the Borel sum of the right-hand side [12-15]. We would like to emphasize that in all these cases Borel summability is obtained a posteriori; by this we mean that one gives first a rigorous definition of the Schwinger functions $S$ in (I.1), generally as the moments of a probability measure on $S^{\prime}\left(\mathbf{R}^{d}\right)$ (functional integral formalism). Then one verifies that the $S$ defined in this way satisfy, as functions of $g$, the hypotheses of one of the standard theorems on Borel summability [20-22]. It is not a completely perturbative proof since it uses the independent definition of $S$ via the functional integral. Therefore the question naturally arises: can perturbative field theory be a fully constructive method, in non-trivial, interesting cases? In particular, if there does not seem to be

1 For a discussion of the relationship of our work to the similar program of 't Hooft [7-10], we refer to [11]; we notice simply here that both methods and perspectives are quite different 
any good definition of $S$ via functional integrals but if one suspects the series in the right-hand side of (I.1) to be Borel summable, is it possible to prove it and to take the Borel sum of the right-hand side of (I.1) as the rigorous definition of $S$ ?

The naive answer to this question would probably be: no. By purely perturbative methods, the Borel transform of the perturbative expansion has been proved to exist near the origin in the Borel plane in rather general cases like $\varphi^{4}$ in any dimension where it is renormalizable $[1,23]$; but this has been accomplished via absolute upper bounds on Feynman amplitudes. To prove Borel summability for nontrivial divergent series made of complicated Feynman amplitudes seems to require such an extremely delicate control of all sorts of cancellations between pieces of the expansion with different signs that it looks almost desperate. Therefore it may be a surprise that as a consequence of this paper at least a partial "yes" can be answered to the above question. What will be shown in the next sections is that if one restricts the summation in (I.2) to planar graphs the right-hand side of (I.1) is a Borel summable power series in dimension 4 and for $g>0$ (asymptotically free case). This result is obtained by a purely perturbative method in which one only looks carefully at the Feynman amplitudes, cuts them into a lot of pieces, and does carefully controlled partial (Borel) resummations of some of these pieces.

We understand that the restriction to planar graphs is an enormous simplification which trims most of the graphs in the original expansion; nevertheless because of the presence of "renormalons" in the planar expansion we expect the series we have Borel-summed to be fully nontrivial ones with 0-radius of convergence (see Sect. IV); moreover the restriction to planarity is not arbitrary; it is motivated by the desire to construct rigorously the large $N$ limit of, say, QCD with gauge group $\mathrm{SU}(N)$. Although the method presented in this paper is not yet able to provide this construction, which involves a control of infrared (or strong coupling) problems too, we think it represents a solution of the ultraviolet problem for planar QCD, and gives some hopes for a complete construction, including massless particles. We would like also to emphasize that large sections of this paper apply to the non-planar case as well; only convergence theorems in Appendix B fail because of the large number of non-planar graphs. This seems an encouraging remark for the study of finite component models with our methods.

The organization of this paper is as follows: in Sect. II we define the model which will be constructed; we also review briefly the recent results on planar models and the motivations to study them. For completeness we recall some of the notions introduced in [1] which are crucial for the rest of the paper. In Sect. III we state our main theorem. We explain how to rewrite the planar expansion in terms of "dressed amplitudes." using the technique of the Appendix in [24]. Also we underline the main unexpected difficulty we met; to bound some special regions of integration in some dressed amplitudes requires a particularly delicate analysis. In Sect. IV we discuss several open problems and possible extensions of our work.

We include a brief discussion of present ideas about the "renormalon" singularities. Finally Appendices A-C are devoted to technical proofs; Appendix A deals with the process of "dressing" in the amplitudes (some earlier examples of this kind of ideas can be found in [19, 24]); Appendices B and C establish the various bounds necessary for the proof of Theorems III.2 and III.3; 
they depend heavily on [1]. The reader unfamiliar with the technicalities of [1] might be mostly interested by this introduction, the first part of Sect. II and Sects. III and IV.

\section{The Planar Model}

\section{II.1. Motivations}

The usual perturbative expansion does not describe correctly the long distance dynamics of non-abelian gauge field theories. It is therefore extremely interesting to develop other approximation schemes for these theories. The most promising ones are the lattice regularization of gauge theories and the $1 / N$ expansion. However it seems that rigorous studies of the continuum limit of lattice theories rely always on some sorts of correlation inequalities (recent works in this area, [25-28] do not escape this rule); most of these correlation inequalities have been only proved for simple spin systems with one or two components, and every attempt to generalize them beyond that range has failed. Therefore the $1 / N$ expansion scheme might be the best proposal to construct rigorously nonabelian gauge theories in the continuum. The interest for this scheme has been triggered by 't Hooft's paper [29], which showed that the first term in the approximation, the large $N$ limit of quantum field theories with a $\mathrm{SO}(N), \mathrm{SU}(N)$ or $\mathrm{U}(N)$ global or local symmetry, is given in perturbation theory by the sum of all the planar amplitudes. Subsequent important works include the study of closed equations for the Wilson loops [30,31], and recently the discovery of the reduction of space-time degrees of freedom which occur in these theories [32-35]. The subject is therefore in vigorous development although up to date there is still no complete construction of what one would like to call for short "planar QCD."

Our construction of the (Borel) sum of the planar diagrams will be done only for a globally symmetric massive model with an asymptotically free interaction; however the method is sufficiently general to be considered also a solution of the ultra-violet problem for general theories of the same kind, including asymptotically free gauge theories (with an infra-red regulator like a finite box with periodic boundary conditions). Therefore we think our work is complementary to the one of Eguchi, Kawai and followers, in which the space-time reduction appears like a solution of the infra-red (infinite volume) problem.

Before giving technical definitions useful for the rest of the paper let us discuss at an elementary level why the planar asymptotically free massive models are the best candidates for a completely perturbative construction in 4 dimensions. The first observation is that in ordinary theories the number of graphs at a given order $n$ grows at least like $n$ !. The singularities that this kind of behavior induces will be called "instanton" singularities to distinguish them from the "renormalons." In ordinary $\varphi^{4}$ theories they presumably prevent the theory from being Borel summable for $g>0$ ("wrong" sign of the coupling constant in our convention). With the usual sign of the coupling constant $(g<0)$, the theory is Borel summable in dimensions 1,2, 3 [12-14]; but this result has never been proved by an analysis staying at the level of Feynman amplitudes and exhibiting the large cancellations which occur between them for $g<0$. Such an analysis seems an extremely difficult 
task because one does not see well graph by graph "where the instanton singularities are"; they are a mean statistical effect. In particular it seems unlikely that one can perform this analysis before one controls completely easier problems such as proving by perturbative methods the Lipatov formulae $[36,37]$ in full detail and for all superrenormalizable dimensions (note that progress has recently been made in this direction [38], which might therefore not be hopeless). If one does not want to deal with instanton singularities and large numbers of graphs, one is quite naturally lead to the planar theories. They are the most interesting for physics in which the number of graphs at order $n$ grows only like an exponential of $n[39,40]$. In superrenormalizable dimensions, since any Feynman amplitude of order $n$ is bounded by a uniform exponential of $n$ [23], it follows immediately that the sum of the planar expansion can be performed at small coupling and is an analytic function of this coupling near the origin ${ }^{2}$. Physically what happened to the instanton singularity in the limit of large number of components is that its action goes to infinity; this stabilizes an otherwise unstable vacuum for the theory with "wrong" sign of $\varphi^{4}$, by preventing tunneling effects to occur with a finite probability.

What makes life more interesting in the 4-dimensional case is that "ultraviolet" singularities almost surely prevent the direct summability of the planar diagrams. We are mostly interested in asymptotically free field theories. It was argued in [4-6] that in this case ultra-violet renormalons should not prevent Borel summability. Since we do not want from the beginning to attack the hard problem of long-distance behavior in gauge theories, we are lead to try first the perturbative construction of asymptotically free massive planar models.

\section{II.2. The Model}

Let us consider a $\mathrm{U}(N)$ massive Hermitian matrix field $\varphi$ in 4-dimensional Euclidean space-time with a $+\operatorname{tr} g \varphi^{4}$ interaction; the connected Schwinger functions for this field are the moments of the following formal measure:

$$
d \mu(\varphi)=Z^{-1} \exp \int d^{4} x \operatorname{Tr}\left\{-\frac{1}{2}\left(\partial_{\mu} \varphi\right)^{2}-\frac{1}{2} m^{2} \varphi^{2}+g \varphi^{4}\right\} \prod_{x} d \varphi(x) .
$$

In (II.1) one should understand $\varphi^{2}$ as $\left(\varphi \cdot \varphi^{*}\right)$ etc. Also (II.1) does not make sense without renormalization. Since our theory is massive, we can fix the renormalization scheme to be the same BPHZ scheme of "subtractions at 0 external momenta" which was used in [1]; it is the most natural for this model. Then after suitable rescaling $(g \rightarrow g / N)$ the perturbative expansion in $g$ of any $\mathrm{U}(N)$ invariant Schwinger function is dominated as $N \rightarrow \infty$ by the planar diagrams [29].

For a Schwinger function with $e$ external momenta there is also a factor $N(1 / N)^{e / 2}$, which means that formally the large $N$ limit of this model is a generalized free field with a complicated two-point function given by the sum of all

2 This result has perhaps not received enough attention; it would be interesting from a mathematical point of view to explore which axioms of axiomatic field theory survive in the planar limit, and in which weak sense they can perhaps be still considered as field theories; this investigation could be done most easily in the superrenormalizable case where one has such a cheap construction of the Schwinger functions as simple sums of convergent series 
planar graphs with two external momenta [41]. It would be nice to analyze rigorously this point in further detail, but in any case our construction will not depend on it.

We introduce now some notations for the perturbative expansion, the Feynman amplitudes in $\alpha$ representation and the renormalization. In fact in the rest of this section we will mostly recall some definitions and some material already contained in [1] which are essential for the rest of the paper.

The connected one-particle irreducible planar Schwinger functions with $e$ external arguments are given by the (for the moment still formal) power expansion in $g$ :

$$
S_{e}(\mathbf{p}, g)=\delta\left(\sum_{q=1}^{e} \mathbf{p}_{q}\right) \sum_{n=0}^{\infty} \frac{(g)^{n}}{n !} \mathbf{a}_{n}^{e}(\mathbf{p}),
$$

where $\mathbf{p}=\left(\mathbf{p}_{1}, \ldots, \mathbf{p}_{e}\right)$ is a set of external Euclidean momenta, and $\mathbf{a}_{n}^{e}$ is the sum of all renormalized Feynman amplitudes associated to 1-P-I planar Feynman graphs with $n$ internal vertices and $e$ external legs. These graphs have exactly 4 lines attached at each vertex. Up to Appendix B, a "graph" will mean a "labeled graph," for which the internal vertices are numbered; this allows a precise discussion of the symmetry factors associated to them and explains the presence of an $n$ ! in (II.2).

\section{II.3. Graph Theory and Feynman Amplitudes}

The following notations are used for any graph $G$ :

$n(G)$ is the number of (internal) vertices of $G$

$l(G)$ is the number of internal lines of $G$,

$e(G)$ is the number of external lines of $G$,

$L(G)$ is the number of independent loops of $G$,

$c(G)$ is the number of connected components of $G$.

$\omega(G)=l(G)-2 L(G)\left(=\frac{e(G)}{2}-2\right.$ if $\left.c(G)=1\right)$ is the superficial degree of convergence of $G$.

We define subgraphs of $G$ which, as in [1], are sets of internal lines of $G$ with the corresponding attached vertices and extend the definitions of $n, l, e, L, c, \omega$ to them in the obvious way. The sign $C$ will always mean strict inclusion; for non-strict inclusion, we use $\subseteq$. By convention an empty sum will always be 0 and an empty product 1 . We also fix the mass $m^{2}$ to be simply 1 in the rest of the paper; it will not be of much concern to us. Since we consider only expansions for truncated Schwinger functions with $e \geqq 2$, and since we chose to subtract the graphs at 0 external momenta, there will be no vacuum $(e=0)$ graphs or subgraphs in our problem, nor graphs containing tadpoles (subgraphs with only one external vertex) [1].

The bare Feynman amplitude $I_{G}$ attached to the graph $G$ is defined in the $\alpha$-parametric representation by the following (possibly divergent) integral:

$$
I_{G}(\mathbf{p})=\int_{0}^{\infty} \ldots \int_{0}^{\infty} \prod_{i=1}^{l} d \alpha_{i} \exp \left(-\sum_{i=1}^{l} \alpha_{i}\right) Z_{G}(\mathbf{p}, \alpha)
$$


The $\alpha$-parametric (or Schwinger) representation (II.3) has a parameter $\alpha$ attached to each internal line $i=1, \ldots, l$ of $G$; the integrand $Z_{G}$ is defined by:

$$
\begin{gathered}
Z_{G}(\mathbf{p}, \alpha)=\frac{\exp \left[-V_{G}(\mathbf{p}, \alpha) / U_{G}(\alpha)\right]}{\left[U_{G}(\alpha)\right]^{2}}, \\
U_{G}(\alpha)=\sum_{S} \prod_{i \notin S} \alpha_{i}, \\
V_{G}(\mathbf{p}, \alpha)=\sum_{T}\left(\prod_{i \notin T} \alpha_{i}\right)\left(\sum_{q \in E_{1}} \mathbf{p}_{q}\right)^{2},
\end{gathered}
$$

where $U_{G}$ and $V_{G}$ are the standard Symanzik polynomials: $S$ runs over the spanning trees (or "one-trees") of $G, T$ over the "two-trees" (spanning trees minus one line) which separate the external lines of $G$ into two non-empty sets, one of which is $E_{1}$. We introduce also the following short notation for the measure in $\alpha$-parametric space:

$$
d \mu\left(\alpha_{i}\right) \equiv \exp \left(-\sum_{i=1}^{l} \alpha_{i}\right) \prod_{i=1}^{l} d \alpha_{i}
$$

\section{II.4. Renormalization}

The subgraphs with $e=2$ ("bipeds") and $e=4$ ("quadrupeds") are superficially divergent and have to be subtracted in (II.3). Following [1] and the earlier references $[42,43]$, we perform the necessary subtractions via an operator acting directly on the integrand $Z_{G}$ of the $\alpha$-parametric representation:

$$
\mathfrak{R}=\sum_{\mathfrak{F}} T_{\mathfrak{F}} ; \quad T_{\mathfrak{F}} \equiv \prod_{F \in \mathfrak{F}}\left(-\mathbf{t}_{F}\right),
$$

where the sum is performed over all closed divergent forests, including the empty one, and $\mathbf{t}_{F}$ is the Taylor subtraction associated to the subgraph $F$. The renormalized amplitude associated to $G$ is then defined as:

$$
I_{G}^{R}(\mathbf{p})=\int_{0}^{\infty} \ldots \int_{0}^{\infty} \prod_{i=1}^{l} d \alpha_{i} \exp \left(-\sum_{i=1}^{l} \alpha_{i}\right) \Re Z_{G}(\mathbf{p}, \alpha) .
$$

For a precise definition of closed divergent subgraphs and forests, and Taylor operators $\mathbf{t}_{F}$ we refer to [1]; we recall that Taylor operators subtract once for a "quadruped" and twice for a "biped," which is quadratically divergent; that a forest is a set of subgraphs which do not overlap, i.e. they are two by two either disjoint or satisfying an inclusion relation of one into the other; that the definition of closed divergent forests is chosen so that all subgraphs in such a forest are superficially divergent and "one-line irreducible" and that "tadpole graphs" do not appear when one reduces one graph in such a forest by the forest. This notion of "reduction" is explained in [1], but let us give again its definition.

For any $\mathfrak{F}$ and $F$ compatible with $\mathfrak{F}$, i.e. such that $\mathfrak{F} \cup\{F\}$ is again a forest, we define:

$$
\begin{gathered}
\mathfrak{I}_{\mathscr{F}}(F)=\left\{F^{\prime} / F^{\prime} \in \mathfrak{F} ; F^{\prime} \subset F ; \text { no } F^{\prime \prime} \text { exists with } F^{\prime} \subset F^{\prime \prime} \subset F\right\} \\
A_{\mathscr{F}}(F)=\bigcup_{F^{\prime} \in \mathfrak{Q}_{\mathfrak{F}}(F)} F^{\prime}=\bigcup_{F^{\prime} \in \mathscr{F}, F^{\prime} \subset F} F^{\prime}
\end{gathered}
$$


Note that $A_{\mathscr{F}}(F)$ may be empty or disconnected and generally does not belong to $\mathfrak{F}$, except if there is exactly one element in $\mathfrak{U}_{\mathfrak{F}}(F)$. Similarly we define $B_{\widetilde{F}}(F)$, the smallest subgraph in $\mathfrak{F}$ which strictly includes $F$. If there is no such subgraph we put $B_{\mathfrak{F}}(F)=G$. If $F \subset F^{\prime}$, the reduced graph $F^{\prime} / F$ is defined by reducing to a single vertex in $F^{\prime}$ every connected component of $F$. The reduction of a subgraph $F$ compatible with a forest $\mathfrak{F}$ by this forest is defined as $F / \mathfrak{F}=F / A_{\mathfrak{F}}(F)$.

In the rest of the paper the word "forest" will always mean "closed divergent forest"; unless otherwise indicated it is assumed that forests called $\mathfrak{F}, \mathfrak{F}^{\prime}, \ldots$, are forests of graphs called $G, G^{\prime}$, and "quadruped" means what is called "closed quadruped" in [1].

\section{II.5. Resummation of Perturbation Theory}

In the study of individual Feynman amplitudes [1], one has to exhibit explicit cancellations between the forests before integrating over $\alpha$ 's in (II.9). In this paper one has to resum infinite sequences of various pieces of Feynman amplitudes to exhibit asymptotic freedom. Both cases can be unified under the abstract point of view of "resummations of perturbation theory," which will be explained now.

Putting together (II.2), (II.8), and (II.9), any given Schwinger function appears as a formal sum over a large space of "contributions." A contribution $\gamma$ is a triplet $(G, \mathfrak{F},[\alpha])$, made of a graph $G$, a forest $\mathfrak{F}$ of $G$, and a set of values $[\alpha] \equiv\left\{\alpha_{i}, i=1, \ldots, l\right\}$ for the parameters of the internal lines of $G$. The value (or the integrand) attached to such a contribution $\gamma$ is defined as:

$$
W(\gamma) \equiv g^{n(G)} \frac{1}{n(G) !} T_{\widetilde{F}} Z_{G}(\mathbf{p}, \alpha) .
$$

The "sum" over contributions means a discrete summation on $G$ and $\mathfrak{F}$, and a continuous integration over $\alpha$ 's with the measure $d \mu(\alpha)$ defined by (II.7).

Formally we write:

$$
S_{e}(\mathbf{p}, g)=\delta\left(\sum_{q=1}^{e} \mathbf{p}_{q}\right) \int_{\Gamma} W(\gamma) d \nu(\gamma),
$$

where $\Gamma$ is the space of all contributions and $d v$ is the appropriate measure.

We never expect absolute convergence of (II.13) and we need therefore a "resummation prescription" to compute it.

Such a resummation prescription is a sequence of "slicings" of the space of all contributions into fibers and base together with the prescription that one should first compute the partial integrals over the fibers, then sum up over the base the values obtained.

This can be formulated precisely in the language of projections (mappings $\varphi$ with $\varphi^{2}=\varphi$ ). A sequence $\varphi_{1}, \ldots, \varphi_{m}$ of projections from $\Gamma$ into itself is called compatible if $\operatorname{Im} \varphi_{i}$, the image of $\varphi_{i}$, is stable by $\varphi_{i+1}$, hence $\varphi_{i}{ }^{\circ} \varphi_{i+1}{ }^{\circ} \varphi_{i}=\varphi_{i+1}{ }^{\circ} \varphi_{i}$. The resummed value of $S_{e}$ according to such a compatible sequence $\varphi_{1}, \ldots, \varphi_{m}$, is defined as the following integral (provided it exists):

$$
\begin{aligned}
S_{e}= & \delta\left(\sum_{q} \mathbf{p}_{q}\right) \int_{\operatorname{Im} \varphi_{m}} d v\left(\gamma_{m}\right) \int_{\operatorname{Im} \varphi_{m-1}} \delta\left(\varphi_{m}\left(\gamma_{m-1}\right)-\gamma_{m}\right) d v\left(\gamma_{m-1}\right) \\
& \cdots \int_{\operatorname{Im} \varphi_{1}} \delta\left(\varphi_{2}\left(\gamma_{1}\right)-\gamma_{2}\right) d v\left(\gamma_{1}\right) \int_{\Gamma} \delta\left(\varphi_{1}(\gamma)-\gamma_{1}\right) W(\gamma) d v(\gamma) .
\end{aligned}
$$


The first basic slicing we have to introduce is the projection $\mathbf{S}$, called in [1] the "classification of forests," which solves the renormalization problem, especially the problem of "overlapping divergences" by exhibiting $I_{G}^{R}$ in (II.9) as a sum of explicitly convergent integrals. Later in this paper we introduce two other projections $\varphi$ and $\psi$, compatible with $\mathbf{S}$, which allows us to exploit asymptotic freedom in a very explicit way. Together with $\mathbf{S}$ they form a resummation prescription in the sense above. The integral (II.14) can be unambiguously defined and will be shown to be the Borel sum of the initial series (II.2).

\section{II.6. The Classification of Forests}

This is our basic tool. Following [1, Sect. III.1], we group the subtraction forests which appear in the definition (II.8) of the $\mathfrak{R}$ operation into appropriate classes. These classes depend on the region in $\alpha$-space that one is considering, so that the cancellations inside each class will make transparent the convergence over the region corresponding to this class.

We introduce a slight improvement of the original definition of these classes in [1]. Let us define the " 2 nd inf," in a finite set of at least two numbers as the element of this set which is immediately greater than the smallest one in this set. Then we replace the technical definition of the quantity $y(F, \mathfrak{F})$ in $[1,($ III.1), p. 79 , items f) and $\mathrm{g})]$ by the simpler definition:

$$
y(F, \mathfrak{F}) \equiv 2 \operatorname{nd} \inf \{j / \sigma(j) \in E\} .
$$

The attentive reader can verify that this new definition does not change anything in any theorems or lemmas in [1], mainly because the technical way in which one compares the quantities associated to different trees in [1, Sect. III.4] always uses the " 2 nd inf" of the indices of the external legs of a subgraph rather than the true infimum.

Let $(G, \mathfrak{F},[\alpha])$ be a contribution. We define a projection

$$
\mathbf{S}:(G, \mathfrak{F},[\alpha]) \stackrel{\mathbf{s}}{\longrightarrow}(G, S(\mathfrak{F}),[\alpha]),
$$

where $S(\mathfrak{I})$ is the "skeleton forest" associated to $\mathfrak{F}$ and defined in $[1]^{3}$, and depends on the value of $[\alpha]$ (in fact only on the Hepp's sector to which $[\alpha]$ belongs [1]). For completeness we will sketch the definition of $S$, introducing useful notations. For any forest $\mathfrak{F}$ and $F \in \mathfrak{F}$, one defines two basic parameters $\alpha(F, \mathfrak{F})$ and $\alpha^{*}(F, \mathfrak{F})$ (the reader might think of them as " $\alpha$ inside $F$ " and " $\alpha$ outside $F$ ", respectively). One has:

$$
\alpha(F, \mathfrak{F}) \equiv \sup _{i \in X(F, \mathfrak{F})} \alpha_{i}
$$

where $X(F, \mathfrak{F})$ is defined precisely in [1], page 78; in fact $X(F, \mathfrak{F})=F / \mathfrak{F}$, except possibly when the presence of bipeds creates technical subtleties. Therefore $\alpha(F, \mathfrak{F})$ is what is called $\alpha_{x(F, \mathscr{F})}$ in $[1] . \alpha^{*}(F, \mathfrak{F})$ is what is called $\alpha_{y(F, \mathfrak{F})}$ in $[1]$, with the modification (II.15) of $y(F, \mathbb{F})$. Hence:

$$
\alpha^{*}(F, \mathfrak{\Im}) \equiv 2 \operatorname{ndinf} \alpha_{i \in E(F, \mathscr{\mho})}
$$

3 By an unfortunate coincidence the word "skeleton forest" was introduced in [1]; it has nothing to do with the "skeleton expansion" considered in [8] or [9] 
where $E(F, \Im)$ is the set of external lines of $F$ internal in $B_{S(\mho)}(F)$. By convention $\alpha^{*}(G, \mathfrak{F})=+\infty$. Notice that the modification (II.15) allows a unified treatment of the cases " $F$ biped" and " $F$ quadruped."

As in [1], we define $S(\mathfrak{F})$ by:

$$
S(\mathfrak{F}) \equiv\left\{F \in \mathfrak{F} / \alpha^{*}(F, \mathfrak{F}) \leqq \alpha(F, \mathfrak{F})\right\} .
$$

The definition, being inductive, starts from the maximal elements of $\mathfrak{F}$. It is proved in [1] that $S$ is a projection, and that:

$$
\alpha(F, \mathfrak{F})=\alpha(F, S(\mathfrak{F})) ; \quad \alpha^{*}(F, \mathfrak{F})=\alpha^{*}(F, S(\mathfrak{F})) .
$$

For a skeleton forest $\mathfrak{F}$ (i.e. $S(\mathfrak{F})=\mathfrak{F}$ ) the structure of the fiber $\left\{\mathfrak{F}^{\prime} / S(\mathfrak{F})=\mathfrak{F}\right\}$ is just $\left\{\mathfrak{F}^{\prime} / \mathfrak{F} \subseteq \mathfrak{F}^{\prime} \subseteq \mathfrak{F} \cup \mathfrak{H}(\mathfrak{F})\right\}$ for some $\alpha$-depending forest $\mathfrak{H}(\mathfrak{F})$. Calling $D_{\mathfrak{F}, \mathfrak{H}}$ the domain of all $\alpha$ 's with $S(\mathfrak{F})=\mathfrak{F}$ and $\mathfrak{H}(\mathfrak{F})=\mathfrak{H}$ (it is a union of Hepp's sectors), the key decomposition of Feynman amplitudes introduced in [1] is:

$$
I_{G}^{R}=\sum_{\overparen{F}, \mathfrak{S}} \int_{D_{\overparen{\mho}, \mathfrak{H}}} d \mu(\alpha) T_{\widetilde{F}} \prod_{H \in \mathfrak{H}}\left(1-t_{H}\right) Z_{G}
$$

Every integral in (II.20) is now absolutely convergent and in contrast with (II.9), can be bounded quite accurately [1].

We introduce also the point of view of "reduction vertices." In this paper we call a "reduction vertex" of a contribution $(G, \mathfrak{F},[\alpha])$ a quadruped of $S(\mathfrak{F})$, namely an element of the forest:

$$
\mathfrak{Q}(\mathfrak{F}) \equiv\{F \in S(\mathfrak{F}) / N(F)=4\}
$$

Since by definition of the Taylor operator $t_{F}$, one has

$$
t_{F} Z_{G}(\mathbf{p}, \alpha)=Z_{F}\left(0, \alpha_{i}, i \in F\right) \cdot Z_{G / F}\left(\mathbf{p}, \alpha_{j}, j \notin F\right)
$$

for any $F$ quadruped, one should indeed imagine that when $F \in \mathfrak{Q}(\mathbb{F})$ the operator $t_{F}$, which is not combined with anything in (II.20) cuts the graph $G$ into the disconnected pieces $F$ and $G / F$, where $F$ is replaced by a "reduction vertex" with 4 lines.

Finally we define, for any (ordinary) vertex $v$ of $G$ the parameter

$$
\alpha^{*}(v, \mathfrak{\mho}) \equiv 2 \operatorname{2ndinf} \alpha_{i},
$$

where $E(v, \mathfrak{F})$ is the set of the lines connected to $v$ which are internal in $B_{S(\mathscr{F})}(v)$, the smallest subgraph of $S(\mathfrak{F})$ which contains $v$. Again one has

$$
\alpha^{*}(v, \mathfrak{F})=\alpha^{*}(v, S(\mathfrak{F}))
$$

When no confusion on the skeleton forest considered is possible, we write simply $\alpha_{F}, \alpha_{F}^{*}, \alpha_{v}^{*}$ instead of $\alpha(F, \mathfrak{F}), \alpha^{*}(F, \mathfrak{F}), \alpha^{*}(v, \mathfrak{F})$.

\section{The Main Steps of the Construction and Results}

Our main theorem states that the planar expansion which forms the right-hand side of (II.2) is Borel-summable as a power series in $g$; its Borel-sum can be taken therefore as the rigorous definition of the left-hand side $S_{e}(\mathbf{p}, g)$. We could obtain in 
fact more than the minimal hypothesis which we verify to apply the "NevanlinnaSokal" theorem [21,22]; it is an easy exercise left to the reader to check that our method gives Watson's theorem [20] in a sector centered around the positive real axis and of opening angle $2 \pi-\varepsilon$ for any $\varepsilon>0$, so that the Borel transform has no singularities in the right half of the complex plane for the Borel-variable; this domain of analyticity is similar to the one found in [10].

Theorem. The planar expansion (II.2), as a power series in the coupling constant $g$, is Borel summable in the usual sense of $[20,22]$.

We would not like to obscure under technicalities the basic ideas used for the construction which allow us to prove this result; they are in fact very natural. Therefore we postpone to Appendices A-C the actual proof of the theorem and in the rest of this section we will only describe these ideas and the main steps of this construction; for an even simpler sketch of these steps we refer to [11].

The starting intuitive idea is that in order for the construction to work one has to exploit asymptotic freedom; there should be an ultra-violet improvement after the bare vertices have been replaced by the (Borel?) sum of the renormalized 4-point amplitudes, and this improvement should lead to convergence.

This is 't Hooft's approach [9] in which the full 4-point function is taken as the running coupling constant. Indeed it is known $[1,8]$ that the sum of planar graphs without divergent subgraphs is an absolutely convergent series. However one has still to prove that the 4-point function behaves as indicated by the renormalization group and that it is the sum of its perturbative series.

In [9], an inductive approach to a fixed point of a set of difference equations is used. The construction is not very practical for computation and its relationship to perturbation theory is somewhat involved in our opinion. Indeed to take the entire 4-point function as a running coupling constant is a "maximal" dressing prescription; in the language of Sect. II.5, it corresponds to an infinite sequence of projections, hence to a huge rearrangement of the initial perturbative series.

From this point of view, the rearrangement of perturbation theory used in this paper is in contrast a "minimal" one, described by only two projections. Staying as close as possible to perturbation theory, we exploit asymptotic freedom inside Feynman amplitudes, without introducing at any stage any global object like the 4-point function. Each step of the construction is completely explicit; therefore our prescription could be used directly for numerical computations. This is possible because asymptotic freedom depends only upon the first coefficient of the $\beta$ function in the Callan-Symanzik equation. This coefficient governs the asymptotic behavior of the $n^{\text {th }}$ order of perturbation theory, e.g. at large external momenta. But this behavior, called the "leading-log" approximation, can also in principle be computed by summing some pieces of the renormalized Feynman amplitudes with $n$ vertices, namely those exhibiting precisely this leading behavior. The only trouble with this approach is that it seems hard to identify all these pieces and to sum them exactly. In [24], we showed not only that this was possible but also that it could be done easily after one has performed the splitting (II.20) of the Feynman amplitudes, precisely into these pieces indexed by "skeleton forests" which solve nicely the renormalization problem. This suggests strongly that this splitting is the truly good way to look at amplitudes. 
Before to go further let us recall the intuitive idea which is at the core of the splitting (II.20). A central problem which prevented for a long time perturbative renormalization from being established on a mathematically sound basis is the problem of "overlapping divergences." Indeed one wants to eliminate the divergences in Feynman amplitudes in a way which is implemented by counterterms in the Lagrangian and therefore respects the basic properties of the theory. One is led to the forest formula (II.9), since it was shown that this formula is nothing but the compact solution of any renormalization which can be implemented by such counter terms. The main mathematical problem has been formulated clearly by several authors (see for instance [46, p. 305]): for complicated renormalized amplitudes it is not clear at all that the integral (II.9) is convergent, because when overlapping divergences are present it seems that some necessary subtractions are missing in (II.9), namely the products $\left(-\mathbf{t}_{F}\right) \cdot\left(-\mathbf{t}_{F^{\prime}}\right)$ when $F$ and $F^{\prime}$ do overlap. The key idea is that one has to perform a Taylor subtraction corresponding to a given subgraph only when one is in a "deep ultraviolet region" for this subgraph, namely when in integration space one tries to integrate the internal momenta of this subgraph truly up to infinity; in other regions it is better not to perform explicitly the $\left(1-\mathbf{t}_{F}\right)$ subtraction corresponding to $F$ on the integrand, but to simply split into the sum of the operator 1 and the operator $-\mathbf{t}_{F}$. If one systematizes this idea one is led to cut the initial region of integration in the amplitudes according to which internal momenta are higher than others, and to regroup the subtractions differently according to which region one is in. In many rigorous works we know of on perturbative renormalization which proves theorems valid to all orders, one finds some form or another of this basic idea [45-50, 43, 1]. In its most systematic and convenient form one splits the integral as completely as possible by ordering all momenta (in $\alpha$-representation, all the $\alpha$ 's). We call "Hepp's sectors" the domains of integration obtained in this way. The classification of forests in [1] is a correct systematization of how one can regroup the subtractions; the "skeleton forests" in a given sector correspond to subgraphs that one should not subtract explicitly in that sector, and conversely the forests we denote by $\mathfrak{H}(\mathfrak{F})$ correspond to subgraphs that one is forced to subtract explicitly [note the factors $\left(-\mathbf{t}_{F}\right)$ for the first ones and $\left(1-\mathbf{t}_{H}\right)$ for the second ones in (II.20)]. As a final remark, we think that a good rule to regroup the subtractions has to be "local" according to the graph: to know whether a given subgraph in a forest should be "skeleton" or not (which means unsubtracted or not) one has to compare the momenta into this subgraph only to the momenta of near-by lines (in our rule [1], the external lines of this subgraph).

We return now to the main problem of computing asymptotic freedom effects directly in the amplitudes. At least since the work of Landau et al. [51-52], it is known that the "leading-log" effects are due to a particular class of graphs, which following them we call "parquet graphs"; they are all obtained by repeated insertions of the same elementary smallest divergent graph $G_{0}=\alpha$, in all possible ways, at any vertex (see Appendix A for a more precise definition). Some parquet graphs are shown in Fig. 1. The important point found in [24] is that within these graphs only very particular pieces are responsible for leading-log behavior, namely these pieces in the splitting (II.20) for which the skeleton forests $\mathfrak{F}$ are maximal among the forests of $G$; each reduced graph $F / \mathfrak{F}$ is then isomorphic to 

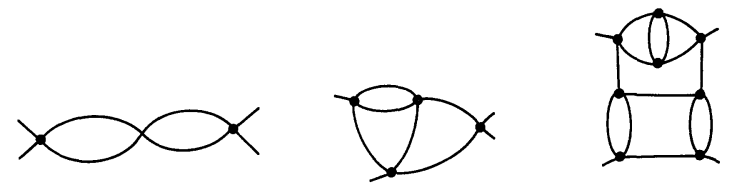

Fig. 1. Parquet graphs

$G_{0}$. Fortunately the presence of the Taylor operators $\left(-\mathbf{t}_{F}\right)$ for $F \in \mathfrak{F}$ is precisely what allows an exact computation of these pieces together with their exact signs [24], because the Taylor operators cut the complicated topological structure of the integrand $Z_{G}$ into a simple factorized product. Not surprisingly, when the precise combinatoric coefficients of every graph are taken into account the final outcome of the computation gives an exact geometric power series in agreement with what the Callan-Symanzik equation indicates if one retains only the first coefficient of the $\beta$ function [24].

The following generalization comes naturally to mind: one should do the same kind of computations in every graph if one wants to exploit for every graph the ultra-violet improvements of asymptotic freedom. This means that for every amplitude, and even more precisely for every contribution $(G, \mathfrak{F},[\alpha])$, one should resum a lot of contributions $\left(G^{\prime}, \mathscr{F}^{\prime},[\alpha]\right)$, obtained from $(G, \mathfrak{F},[\alpha])$ by inserting at any vertex of $G$ an arbitrary parquet subgraph and by integrating over the regions for which some maximal forest in these parquet subgraphs (called a "parquet forest") is "skeleton"; in this way one can transform the original expansion (II.2) into a "renormalization group improved" expansion of the same type, with the bare vertices replaced by dressed ones which explicitly display ultraviolet improvement. These dressed vertices depend of the $\alpha$-parameters of their incoming lines, which is no surprise because the condition for a subgraph to be "skeleton" explicitly depends on the values of the parameters of the external lines of this subgraph. The ultra-violet improvement gained at every vertex makes the "dressed" planar expansion absolutely convergent. One understands in a transparent way why the whole process is a Borel summation rather than a simple one; it is because the ratio of the geometric power series summed at each vertex in the dressing process can be greater than 1 in some regions of integration in the $\alpha$ space. Hence the "dressing operation" (and the whole construction) is not an absolutely convergent summation. In particular it fails for the opposite sign of the coupling constant: the geometric power series are not alternate any more, and their sum display explicitly poles (the Feldman-Landau ghosts) over which integration is impossible.

In the realization of this program, we met an unexpected difficulty. The dressing process that we define uses only some regions of the parquet graphs. Therefore the dressed expansion still contains regions of integration for arbitrarily large parquet graphs. In contrast with every other contribution to the dressed expansion, these terms are hard to bound by a convergent series. For them, we use a rather inelegant and complicated mixture of arguments from the $\alpha$ - and momentum representation (see Appendix B). There does not seem to be any analog of this problem in 't Hooft's construction [9]. 
The attentive reader could wonder whether it is possible to resum enormous alternate pieces and get extremely small factors at each vertex, which certainly is sufficient to make the dressed sum convergent in a rather trivial fashion. The answer is that this is certainly possible. This remark is not deep and is only related to the rather trivial fact that divergent series with terms of arbitrary signs can be rearranged to give almost any answer one wants. The only thing which one cannot do anymore if one makes too large partial resummations is to prove that the sum of the overdressed series obtained in this way is the Borel sum of the initial expansion; that is to show uniform bounds on the Taylor remainder of this sum (Theorem III.3 below). Hence there are two constraints on the dressing process: one should dress enough in order for the dressed expansion to converge (Theorem III.2) and not too much in order for the result to be related unambiguously to the initial series (Theorem III.3). As a result of this paper, these two constraints can be satisfied simultaneously in the planar case.

To summarize this discussion, we think that Theorem III.3 below is the cornerstone which controls that there is no arbitrariness in our construction.

We decompose the theorem above in a sequence of three intermediate results. The first step is the dressing process. Using the language of Sect. II.5, we define two projections $\varphi$ and $\psi$ on the set $\Gamma$. They correspond respectively to the dressing of ordinary vertices and of "reduction vertices." It is in fact sufficient to define $\psi$ only on the image of $\varphi$, called by definition the set of "intermediate contributions." The image of $\psi$ will be called the set of "fundamental contributions":

$$
\Gamma \stackrel{\varphi}{\longrightarrow} \text { intermediate contributions } \stackrel{\psi}{\longrightarrow} \text { fundamental contributions. . }
$$

The precise definition of $\varphi$ and $\psi$ is given in Appendix A. The following theorem allows us to resum exactly the contributions in the "fiber" mapped by $\psi \circ \varphi$ onto a given fundamental contribution:

Theorem III.1 (Dressing Process). For any fixed intermediate contribution $\gamma=(G, \mathfrak{F},[\alpha])$, with the notations of (II.14), one has

$$
\int_{\Gamma} \delta\left(\varphi\left(\gamma^{\prime}\right)-\gamma\right) W\left(\gamma^{\prime}\right) d v\left(\gamma^{\prime}\right) \equiv W(\gamma) \operatorname{OD}_{G}^{\widetilde{F}}(\alpha) \equiv I(\gamma),
$$

and for any fixed fundamental contribution $\gamma=(G, \mathfrak{F},[\alpha])$,

$$
\begin{gathered}
\int_{\operatorname{Im} \varphi} \delta\left(\psi\left(\gamma^{\prime}\right)-\gamma\right) I\left(\gamma^{\prime}\right) d v\left(\gamma^{\prime}\right) \equiv W(\gamma) D_{G}^{\mathfrak{f}}(\alpha) \\
\equiv J(\gamma) \equiv J_{G}^{\mathfrak{F}}(\alpha) \equiv I(\gamma) \operatorname{RD}_{G}^{\mathfrak{F}}(\alpha) .
\end{gathered}
$$

The summations in (III.1) and (III.2) are absolutely convergent for $g$ sufficiently small, depending on the $\alpha$-parameters in $\gamma$. The result of these summations, by definition, is expressed by the two multiplicative factors $\mathrm{OD}_{G}^{\mathbb{F}}(\alpha)$ and $\mathrm{RD}_{G}^{\mathscr{F}}(\alpha)$ (for "ordinary" and "reduction" dressing). Together they form the dressing factor D $D_{G}^{\Im}(\alpha)$ of a fundamental contribution:

$$
D_{G}^{\widetilde{\Im}}(\alpha) \equiv \mathrm{OD}_{G}^{\widetilde{f}}(\alpha) \cdot \mathrm{RD}_{G}^{\widetilde{J}}(\alpha) .
$$


Moreover, $\mathrm{OD}$ and $\mathrm{RD}$ are factorized, respectively over the ordinary and the "reduction" vertices of $(G, \mathfrak{F},[\alpha])$ :

$$
\begin{gathered}
\operatorname{OD}_{G}^{\mathscr{F}}(\alpha)=\prod_{v \in G} \operatorname{od}\left(\alpha_{v}^{*}\right), \\
\operatorname{RD}_{G}^{\widetilde{F}}(\alpha) \prod_{F \in \mathbb{Q}(\mathscr{\xi})} \operatorname{rd}\left(\alpha_{F}, \alpha_{F}^{*}\right),
\end{gathered}
$$

where $\alpha_{v}^{*}, \alpha_{F}, \alpha_{F}^{*}$ and $\mathfrak{Q}(\mathfrak{F})$ have been defined in Sect. II.6.

The proof of Theorem III.1 is in Appendix A, together with an explicit definition of the functions od and rd in (III.4) and (III.5). Let us remark that by (II.19) and (II.24):

$$
D_{G}^{\mathfrak{F}}(\alpha)=D_{G}^{S(\mathfrak{F})}(\alpha) .
$$

For $\alpha$ small, od $(\alpha)$ behaves as $[1+g \cdot \operatorname{const}|\log \alpha|]^{-1}$ (A.13)-(A.15). Hence it realizes the logarithmic ultraviolet improvement characteristic of asymptotic freedom. The "reduction" dressing factor RD is a technical complication, somewhat related to the problem of exceptional momenta in momentum space, which is unfortunately necessary to our proofs.

If some $\alpha$ 's in $\gamma$ are smaller than $\exp \left(-\frac{\text { const }}{g}\right)$, the sums in (III.1) and (III.2) may no longer converge absolutely, but the factor $D_{G}^{\widetilde{\mho}}(\alpha)$ still makes sense as the unique analytic continuation in $g$ of the corresponding factor defined at very small $g$. Equations (III.1) and (III.2) hold in this case in this weaker sense.

Returning to the resummation program, one should sum now over all fundamental contributions $\gamma$ the dressed integrand $J(\gamma)$. However, even in the dressed expansion one should perform cancellations between forests (i.e. renormalize) before integrating over $\alpha$ 's. For instance quadratic divergence (mass renormalizations) are certainly not screened by the logarithmic improvement in the dressing factor and have to be correctly subtracted. In fact, we tailor-made the projections $\varphi$ and $\psi$ to be compatible with the classification of forests, so that the dressed expansion can be renormalized as easily as the ordinary one. This is obtained first by constructing dressing factors which depend only on $S(\mathfrak{F})$ (III.6), and second, by ensuring that no counterterm was used for dressing in regions where in (II.20) it has to be combined in a renormalization. This is expressed precisely by:

Lemma III.1. $(G, \mathfrak{F},[\alpha])$ is fundamental if and only if $(G, S(\mathfrak{F}),[\alpha])$ is fundamental.

Proof. It follows trivially from the definition of fundamental contributions in Appendix A.

Let us call $\Delta_{\mathfrak{y}}$ the domain of all $\alpha$ 's such that $(G, \mathfrak{F},[\alpha])$ is fundamental. We define also the domain $\Delta_{\mathfrak{F}, \mathfrak{H}} \equiv D_{\mathfrak{F}, \mathfrak{h}} \cap \Delta_{\mathfrak{F}}$, where $D_{\mathfrak{\mho}, \mathfrak{H}}$ is defined in Sect. II.6. The following theorem tells us in which precise way the dressed expansion is renormalized and resummed.

Theorem III.2 (Convergence of the Dressed Expansion). For any graph $G$ and forests $\mathfrak{F}$ and $\mathfrak{H}$ of $G$ the integral

$$
J_{G}^{\mathfrak{F}, \mathfrak{H}}(\mathbf{p}) \equiv \int_{\Delta \mathfrak{F}, \mathfrak{S}} d \mu(\alpha) \sum_{\widetilde{F} \subseteq \mathcal{A} \subseteq \mathscr{F} \cup \mathfrak{S}} J_{G}^{\mathcal{S}}(\mathbf{p}, \alpha)
$$


is absolutely convergent. Moreover the series

$$
S_{e}(\mathbf{p}, g)=\delta\left(\sum \mathbf{p}_{q}\right) \sum_{G} J_{G}(\mathbf{p})\left(\text { with } J_{G}(\mathbf{p}) \equiv \sum_{\mathfrak{F}, \mathfrak{H}} J_{G}^{\mathfrak{F}, \mathfrak{H}}(\mathbf{p})\right)
$$

is absolutely convergent for any $g$ complex with $\operatorname{Re} g>0$ and $|g|$ sufficiently small. Its sum, $S_{e}(\mathbf{p}, g)$, is analytic in that domain.

In(III.8) the sum is of course performed over all planar graphs $G$ with $e$ external lines. Note that (III.8) is not a power series in $g$, because of the $g$-dependence of $D_{G}^{\widetilde{r}}$, hence of $J_{G}$. We do not try to find the best possible domains of convergence, or its dependence with the external momenta p; but our method gives uniform estimates in $\mathbf{p}$ for the domain of analyticity in $g$, as expected from asymptotic freedom.

The proof of Theorem III. 2 is in Appendix B. Note that by Lemma III.1 and by (II.20), in (III.7) and (III.8) every fundamental dressed contribution to $S_{e}$ has been summed exactly once, completing the program of (II.14).

The crucial question is: is it legitimate to do the summation in this way, and is the object $S_{e}$ we constructed really related to the planar expansion considered as a power series in $g$ ? This question is answered by the next theorem.

Theorem III.3 (Borel Summability). There exists a constant K, such that for $\operatorname{Re} g>0$ and $|g|<\varepsilon, \varepsilon$ fixed sufficiently small one has for any $k$ :

$$
\left|S_{e}(\mathbf{p}, g)-\sum_{G / n(G) \leqq k-1} \frac{g^{n(G)}}{n(G) !} I_{G}^{R}(\mathbf{p})\right| \leqq k !(K|g|)^{k},
$$

where the sum in (III.9) is performed over all renormalized amplitudes with at most $k-1$ vertices and exactly e external lines.

This theorem is proved in Appendix C. At once, inequality (III.9) tells us that the object $S$ that we constructed is $C^{\infty}$ at $g=0$ that its Taylor series at $g=0$ is exactly the renormalized planar expansion, that this expansion is Borel summable, and that $S$ is its correct Borel sum! [21,22]. Therefore it answers fully the question above. We remark that to establish (III.9) we need again the combinatoric machinery developed in [1] to bound renormalized Feynman amplitudes (see Appendix C).

\section{Open Problems}

In this section we list some of the problems which might be attacked with our methods. As we said in the introduction, we propose to analyze in detail the "renormalon" singularities within the planar expansion, which is the $N \rightarrow \infty$ limit of matrix models. In fact the first reference we know of which describes the large factorial behavior of 4-dimensional renormalized Feynman amplitudes is [2], where this behavior was discovered also in the context of an $N \rightarrow \infty$ limit but for a $N$-component vector model (for earlier investigations, in field theory, of nonanalytic behavior in the coupling constant due to 4-dimensional ultra-violet problems, see $[53,54])$. Further studies [3-6] realized the generality of the phenomenon. It was argued in [4] that the large amplitudes create singularities ("renormalons") in the complex plane associated to the Borel transform of the ordinary expansion. 
One should notice that one is still far from any proof of these heuristic arguments. The only case where this "renormalon" conjecture can be proved is precisely in the $N \rightarrow \infty$ limit of a vector model with $N$ components. But these models are rather trivial; in the case of a $\varphi^{4}$ interaction they correspond only to the summation of "chains of bubbles" and are explicitly solvable. In the models with finite $N$ there is a balance between the number of graphs which have an amplitude of a given size and this size [1], so that it would require an extremely fine analysis to prove that there is no conspiracy of a large number of small amplitudes to kill the effects due to a small number of large amplitudes. Our proposal to study and prove the conjectures about existence, position, structure etc.... of the renormalons in planar expansion is an intermediate one; the corresponding problems are neither trivial like in the $N \rightarrow \infty$ limit of vector models, nor extremely hard as in the full expansion. They appear solvable because conspiracies of a large number of small amplitudes cannot occur in the planar world which has only a few graphs.

Before we go on let us explain why we consider the detailed study of renormalon singularities worth the trouble, even in the simplified planar case.

For a theory which is not asymptotically free, like the ordinary $-g \varphi_{4}^{4}$ theory, the renormalons should stand on the positive real axis of the Borel plane, preventing ordinary Borel summability. These singularities, which are clearly related to the "Landau argument" [51,52], are responsible for the (apparent?) triviality of $\varphi_{4}^{4}$. Therefore it should be possible to connect them to the rigorous lattice inequalities found recently [25-27]. These inequalities fall short from proving the triviality of any continuum limit of lattice theories with an ordinary $\varphi_{4}^{4}$ action and nearest-neighbor interaction. What seems still to be lacking in the approaches [25-27] is a control of the logarithmic factors which are responsible for triviality in dimension 4 . This control might be provided by an analog of the resummation technique presented in this paper, adapted to the lattice situation. In our opinion it might be the same problem as the proof of existence of ultraviolet renormalons in the full expansion of $\varphi_{4}^{4}$.

Let us return to the planar case. The easiest result we think could be derived by our methods is a rigorous proof of the divergence of the planar expansion, perhaps in the style of the proof for $\varphi_{3}^{4}$ [19]. This would be interesting because one would like to be sure that the complicated Borel resummation constructed in this paper has not been applied just to a common convergent series! Also since there is not a large number of graphs in the theory, a proof of its divergence is already an indication that "renormalons" do exist.

More generally, we are encouraged in the study of non-perturbative effects induced by planar renormalons by recent studies. In the $1 / N$ expansion for vector models, the existence, location and structure of the infrared and ultraviolet renormalons has been found [56], together with the analytic continuation of the modified Borel transform [55] around them. The prescription of summation in the modified-Borel-transform plane are non-unique. This sheds new light on the meaning of the operator product expansion $[57,58]$, and on the triviality problem for $\varphi_{4}^{4}$ [59]. We consider the planar world a good laboratory for a complete version of these results.

Technically we believe also that it might be interesting to find an analog in $\alpha$-parametric space of the "quenched momenta" prescription for planar perturbative expansions [35] in order to bring our methods closer to the ones developed in 
[32-35]. Because dimensional interpolation of amplitudes in $\alpha$ space is easy, this might lead to a rigorous understanding of the $\varepsilon$ expansion around $d=4$. An interesting problem would be in particular the study of the ultra-violet stable fixed point near the origin in $4+\varepsilon$ dimensional planar models. As previously noticed, one has an easy construction below $d=4$ [23], in contrast with the full non-planar models which have not yet been defined rigorously in complex non-integer dimension, even in super-renormalizable cases.

Let us emphasize the most promising aspect of this work: every estimate in this paper applies without any change to non-planar amplitudes; only the numerical value of the first coefficient of the $\beta$-function changes. Therefore from Theorem III.1 and Lemma B.1 one gets:

Theorem IV.1. For any unlabeled graph $G$, planar or not, the dressed amplitude $K_{G}(\mathbf{p}, g)$ is bounded, for $\operatorname{Re} g>0$ and $|g|<\varepsilon$ by:

$$
\left|K_{G}(\mathbf{p}, g)\right| \leqq[K(\varepsilon)]^{n(G)}
$$

for a fixed $K(\varepsilon)$, with $\lim _{\varepsilon \rightarrow 0} K(\varepsilon)=0$.

A behavior like (IV.1) is characteristic of superrenormalizable theories $([60,23])$. Therefore our dressing process exhibits the deep similarity between superrenormalizable and renormalizable asymptotically free theories. Only the summation in Theorem III.2 fails in the non-planar case, because of the large number of graphs involved.

We think that putting our resummation prescriptions and Theorem IV.1 together with the solution of superrenormalizable theories due to Glimm and Jaffe (phase space expansion [60]), one should be able to construct any stable, massive and asymptotically free renormalizable theory. Unfortunately there is no simple theory of this kind in 4 dimensions.

Finally we mention as immediate by-products of this paper the Borel summability of various approximations to massive AF theories, obtain by retaining at most (const) ${ }^{n}$ graphs of order $n$; for instance the sum of amplitudes with a fixed number of "handles" (higher terms in the $1 / N$ expansion) or the "parquet approximation" [52], in which one retains all parquet graphs, including non-planar ones. This last case involves graphs with an unbounded number of handles: therefore the corresponding result probably does not follow from [7-10].

\section{Appendix A: The Dressing Process}

We will define precisely the projections $\varphi$ and $\psi$ introduced in Theorem III.1, solve the combinatoric problem of performing exactly the sums in (III.1) and (III.2), and give simple estimates for the dressing functions od and $\mathrm{rd}$.

We recall the notion of a parquet graph and of parquet forests (called "complete forests" in [24]).

Definition $A$.1. A parquet graph is a quadruped $G$ such that there exists a forest $\mathfrak{F}$ in $G$ with $n(G)-1$ elements. Any such forest will be called a parquet forest of $G$. 
It is easy to check that if $\mathfrak{F}$ is a parquet forest, $F / \mathfrak{F}$ is isomorphic to $G_{0}=\varnothing$ for every $F \in \mathfrak{F}$. Therefore one can generate all the parquet graphs at order $n$ by replacing any arbitrary vertex in any parquet graph of order $n-1$ by $G_{0}$, in all possible ways. If $G$ is a parquet graph and $\mathfrak{F}$ a parquet forest of $G$, any $F / \mathfrak{F}$ has exactly two internal lines, as $G_{0}$ itself, and if $F \neq G$, there are exactly two external lines of $F$ which are internal in $B_{\overparen{f}}(F)$ and they are all the lines of $B_{\overparen{\mho}}(F)$. We define also in the obvious way the notions of parquet subgraphs and subforests.

Definition A.2. A contribution $(G, \mathfrak{F},[\alpha])$ is "intermediate" if and only if there is no $F$ in $S(\mathfrak{F})$ isomorphic to $G_{0}$.

Remark that if $F$ in $\mathfrak{F}$ is isomorphic to $G_{0}$ the condition $F \in S(\mathfrak{F})$ is just:

$$
\alpha(F, \mathfrak{F})=\sup _{i \in F} \alpha_{i} \geqq \alpha^{*}(F, \mathfrak{F}) .
$$

The following equivalent definition is useful:

Proposition A.1. A contribution $(G, \mathfrak{F},[\alpha])$ is intermediate if and only if there does not exist any parquet subgraph $G^{\prime}$ in $G$ with a parquet forest $\mathscr{F}^{\prime}$ of $\mathfrak{F}$ such that $\mathfrak{F}^{\prime} \leqq S(\mathfrak{F})$. In other words, there does not exist any parquet subgraph $G^{\prime}$ in $G$ with a forest $\mathfrak{F}^{\prime}$ of $G^{\prime}$ which is a subforest of $\mathfrak{F}$, contains $G^{\prime}$, is such that any $F / \mathfrak{F}^{\prime}$ is isomorphic to $G_{0}$ if $F \in \mathfrak{F}^{\prime}$, and verifies:

- for

$$
F \in \mathscr{F}^{\prime}, \quad F \neq G^{\prime}, \quad \sup _{i \in F / \mathscr{F}^{\prime}} \alpha_{i} \geqq \sup _{i \in B \mathscr{F}^{\prime}(F) / \mathscr{F}^{\prime}} \alpha_{i},
$$

- and

$$
\sup _{\left.i \in G^{\prime}\right\urcorner \mathscr{F}^{\prime}} \alpha_{i} \geqq \alpha^{*}\left(G^{\prime}, \mathfrak{F}\right) \text {. }
$$

Indeed if $B_{\mathbb{F}^{\prime}}(F) / \mathscr{F}^{\prime}$ is isomorphic to $G_{0}$, it is easy to verify that

$$
\alpha^{*}\left(F, \mathfrak{F}^{\prime}\right)=\sup _{i \in B \mathfrak{F}^{\prime}(F) / \mathscr{F}^{\prime}} \alpha_{i}
$$

To define $\varphi$, consider a contribution $\left(G^{\prime}, \mathfrak{F}^{\prime},\left[\alpha^{\prime}\right]\right)$. One identifies the maximal parquet subgraphs $G_{1}^{\prime}, \ldots, G_{k}^{\prime}$ and parquet subforests $\mathfrak{F}_{1}^{\prime}, \ldots, \mathfrak{F}_{k}^{\prime}$ of $G_{1}^{\prime}, \ldots, G_{k}^{\prime}$ which belong to $\mathscr{F}^{\prime}$ and satisfy (A.2) and (A.3). The existence of such a maximal family follows from the requirement $\mathfrak{F}_{i}^{\prime} \subset \mathfrak{F}^{\prime}$, and the fact that $\mathfrak{F}^{\prime}$ is a forest. We define $\varphi$ by:

$$
\begin{gathered}
\varphi\left[\left(G^{\prime}, \mathscr{F}^{\prime},[\alpha]\right)\right] \equiv(G, \mathfrak{F},[\alpha]), \quad G \equiv G^{\prime} / \bigcup_{j=1}^{k} G_{j}^{\prime}, \\
\mathfrak{F} \equiv \mathfrak{F}^{\prime} / \bigcup_{j=1}^{k} \mathfrak{F}_{j}^{\prime}, \quad[\alpha] \equiv\left[\alpha_{i}^{\prime}, i \in G^{\prime} / \bigcup_{j=1}^{k} G_{j}^{\prime}\right] .
\end{gathered}
$$

The notation in (A.4) means that $\mathfrak{F}$ is the forest of $G$ obtained from $\mathfrak{F}^{\prime}$ in the natural way; one suppresses the subgraphs in $\bigcup_{j=1}^{k} \mathfrak{F}_{j}^{\prime}$ and the other subgraphs of $\mathfrak{F}^{\prime}$ are reduced by $\bigcup_{j=1}^{k} G_{j}^{\prime}$. $[\alpha]$ is obtained from $[\alpha]$ by simply deleting the parameters of the internal lines which are reduced, hence belong to $\bigcup_{j=1}^{k} G_{j}^{\prime}$ (see Fig. 2). 


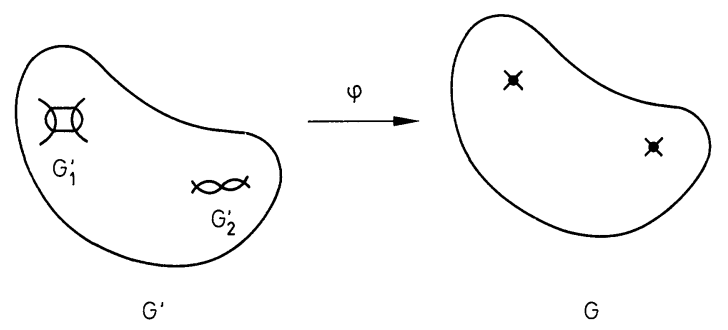

Fig. 2. The mapping $\varphi$ on graphs

It is easy but very important to check that $\varphi$ is a projection, namely that $\varphi^{2}=\varphi$.

We come now to the actual proof of (III.1). Let us consider a contribution $\left(G^{\prime}, \mathfrak{F}^{\prime},[\alpha]\right)$ for which $\varphi\left[\left(G^{\prime}, \mathfrak{F}^{\prime},\left[\alpha^{\prime}, \alpha\right]\right)\right]=(G, \mathfrak{F},[\alpha])$. The vertices of $G, v_{1}, \ldots, v_{n}$ are the reduction vertices of corresponding parquet subgraphs $G_{1}^{\prime}, \ldots, G_{n}^{\prime}$ in $G^{\prime}$, similar to the subgraphs $G_{1}^{\prime}, \ldots, G_{k}^{\prime}$ considered in (A.4) except that some of them (possibly all of them if $\left(G^{\prime}, \mathfrak{F}^{\prime},[\alpha]\right)=(G, \mathfrak{F},[\alpha])$...) can be simply a single vertex. Similarly there are (possibly empty) subforests $\mathfrak{F}_{1}^{\prime}, \ldots, \mathfrak{F}_{n}^{\prime}$ of $\mathfrak{F}^{\prime}, \mathfrak{F}_{j}^{\prime}$ being a parquet forest of $G_{j}^{\prime}$.

Let $k(j)$ be the number $(\geqq 1)$ of internal vertices of $G_{j}^{\prime}$. We number the elements of $\mathscr{F}_{j}^{\prime}$ as $F_{j}^{1}, \ldots, F_{j}^{k(j)-1} \equiv G_{j}^{\prime}$, in a way which is compatible with the partial ordering of $\mathfrak{F}_{j}^{\prime}$ induced by inclusion; hence one has

$$
F_{j}^{p} \subset F_{j}^{q} \Rightarrow p<q .
$$

For $p=1, \ldots, k(j)-2$ let us call $b(p)$ the index of $B_{\widetilde{\mho}_{j}^{\prime}}\left(F_{j}^{p}\right)$. We call $\alpha_{j}^{p}$ the 2 nd inf (or the sup ...) of the parameters of the two lines of $F_{j}^{p} / \mathscr{\mho}_{j}^{\prime}$, which is isomorphic to $G_{0}$; the parameter of its other line will be called $\beta_{j}^{p} \alpha_{j}^{p}$; we have of course $0 \leqq \beta_{j}^{p} \leqq 1$. Finally we put $\alpha_{j} \equiv \alpha^{*}\left(G_{j}^{\prime}, \mathfrak{F}^{\prime}\right)=\alpha^{*}\left(v_{j}, \mathfrak{F}\right)$ and $n^{\prime} \equiv n\left(G^{\prime}\right)$. Note that $\alpha_{j}^{p}=\alpha\left(F_{j}^{p}, \mathfrak{F}^{\prime}\right)$. The left-hand side of (III.1) can be written as:

$$
\begin{aligned}
& \sum_{G^{\prime}, \mathscr{F}^{\prime}} \int_{\left\{\begin{array}{c}
\alpha_{j}^{b(p)} \leqq \alpha_{j}^{p}<\infty, p=1, \ldots, k(j)-2 \\
j_{j} \leqq \alpha_{j}^{k}(j)-1, \ldots \infty \\
0 \leqq \beta_{j}^{p} \leqq 1, p=1, \ldots, k(j)-1
\end{array}\right.} \frac{g^{n^{\prime}}}{n^{\prime} !} \sum_{j=1}^{n}\left\{\prod_{p=1}^{k(j)-1} 2 \alpha_{j}^{p} d \alpha_{j}^{p} d \beta_{j}^{p}\right. \\
& \left.\ldots\left[\prod_{p=1}^{k(j)-1} \exp \left[-\alpha_{j}^{p}\left(1+\beta_{j}^{p}\right)\right]\right] T_{\widetilde{\mho}_{j}^{\prime}}\right\} T_{\widetilde{\mho}} Z_{G^{\prime}}\left(\mathbf{p}, \alpha^{\prime}\right) .
\end{aligned}
$$

The factor 2 in front of $\alpha_{j}^{p}$ is present because there are two values of the parameters of the internal lines of $F_{j}^{p}$ corresponding to the same set of values for $\alpha_{j}^{p}, \beta_{j}^{p}$. To evaluate the complicated integral (A.6) let us remark that, iterating (II.22), the $T_{\mathbb{F}_{j}^{\prime}}$ operator factorizes the integrand $Z_{G^{\prime}}$ into $Z_{G}\left(\mathbf{p}, \alpha_{i}\right) \prod_{j=1}^{n} \prod_{p=1}^{k(j)-1}\left[\alpha_{j}^{p}\left(1+\beta_{j}^{p}\right)\right]^{-2}$ (we used the value of $U_{G_{0}}=\alpha_{1}+\alpha_{2}$ ). The integrals over the parameters $\alpha_{j}^{p}$ and $\beta_{j}^{p}$ factorize in (A.6) and they can be done almost explicitly. Let us define:

$$
\xi(x) \equiv \int_{x}^{\infty} \frac{d \alpha}{\alpha} \int_{0}^{1} \exp [-\alpha(1+\beta)](1+\beta)^{-2} 2 d \beta .
$$


It is not hard to verify that, for any value of $j$ in (A.6):

$$
\begin{aligned}
& \underset{\substack{\alpha_{j}^{b(p)} \leqq \alpha_{j}^{p}<\infty, p=1, \ldots, k(j)-2 \\
\alpha_{j} \leqq \alpha_{j}^{k(j)-1 \leqq \infty} \\
0 \leqq \beta_{i}^{p} \leqq 1, p=1, \ldots k(j)-1}}{k \prod_{p=1}^{k(j)-1}}\left(\frac{d \alpha_{j}^{p}}{\alpha_{j}^{p}}\right)\left(2 d \beta_{j}^{p}\right) \exp \left[-\alpha_{j}^{p}\left(1+\beta_{j}^{p}\right)\right]\left(1+\beta_{j}^{p}\right)^{-2} \\
& \quad=\frac{1}{[k(j)-1) !} C\left(\widetilde{F}_{j}^{\prime}\right)\left[\xi\left(\alpha_{j}\right)\right]^{k(j)-1},
\end{aligned}
$$

where $C\left(\mathfrak{F}_{j}^{\prime}\right)$ is the number of possible orderings of $\mathfrak{F}_{j}^{\prime}$ which are compatible with inclusion in the sense of (A.5). Indeed for every ordering $\sigma$ of $\mathfrak{F}_{j}^{\prime}$, the integral

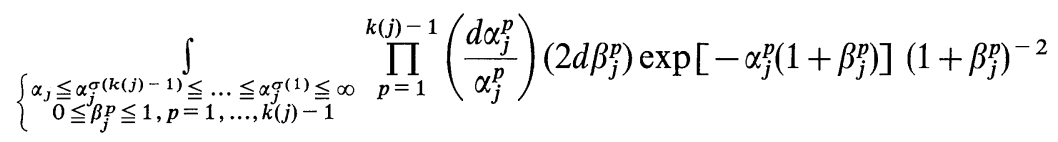

obviously does not depend on $\sigma$; therefore it is equal to

$$
\frac{1}{[k(j)-1] !}\left[\xi\left(\alpha_{j}\right)\right]^{k(j)-1} .
$$

Now we cut the domain of integration in (A.8) into pieces associated to complete orderings of the parameters $\alpha_{j}^{p}$. The number of such pieces compatible with the constraints in (A.8) is precisely $C\left(\widetilde{F}_{j}^{\prime}\right)$, and the integration over any of these pieces gives (A.9), hence (A.10). This achieves the proof of (A.8).

The proof of (III.1) is now reduced to a purely combinatoric problem albeit a non-trivial one. The following key lemma controls the combinatoric coefficients (symmetry factors) associated to the dressing contributions; it is the analog of Lemma A.9 in [24], but we have to be careful about the restriction to planar graphs which change the counting problem.

Lemma A.2. Let $(G, \mathfrak{F},[\alpha])$ be an intermediate contribution. We want to count the number of triplets $\left(G^{\prime}, \widetilde{F}^{\prime}, \sigma\right)$, where as before $G^{\prime}$ is obtained from $G$ by inserting $G_{j}^{\prime}$ at the vertex $v_{j}$ of $G, \mathfrak{F}^{\prime}$ is the union of the $\mathfrak{F}_{j}^{\prime}$ and $\sigma=\left(\sigma_{j}\right), j=1, \ldots, n$ is a collection of orderings of $\widetilde{F}_{j}^{\prime}$ which in each $\mathfrak{F}_{j}^{\prime}$ are compatible with inclusion. We claim this number is nothing but

$$
\frac{n^{\prime} !}{n !} \prod_{j=1}^{n}\left\{c^{k(j)-1}[k(j)-1] !\right\}
$$

where $c=8$. (In the non-planar case the value of $c$ would be 36.)

Proof. We follow the proof of Eq. (A.27) in [24], except for one important change, the cyclic ordering of the lines around a vertex. To count Wick contractions it is convenient to define the mapping $\varphi$ at the level of "ordered contributions" which will be defined below. They are "sub-contributions" in the sense that in general a contribution is a sum of several "ordered contributions"; accordingly the formulation of Sect. II.5 should not be taken too literally ${ }^{4}$.

4 We thank E. Speer for pointing out this counting subtlety to us 
Let $G^{\prime}$ be a labeled graph. The half lines attached to a vertex $i$ are called $i_{1}, i_{2}, i_{3}$, $i_{4}$, following the natural cyclic ordering. An "ordered contribution" corresponds to the choice of an arbitrary order for the vertices of each contracted $G_{j}^{\prime}$ in $G^{\prime}$. Hence there are $\prod_{j} k(j)$ ! ordered contributions associated to a given contribution, each with a weight $[\Pi k(j) !]^{-1}$. To specify $\varphi$, we construct $G$ from $G^{\prime}$ by the following algorithm. One considers the minimal subgraph in an $\mathfrak{F}_{j}^{\prime}$ according to the order $\sigma_{j}$. It is an elementary $G_{0}$ subgraph, with vertices numbered $i$ and $j$. We contract it to a reduction vertex named $i$ or $j$ according to which of them is minimal in the arbitrary order chosen. Then we delete the other number and rename the vertices of $G^{\prime}$ from 1 to $n^{\prime}-1$, respecting the order. There is also a simple rule of how to attribute the half lines of the reduction vertex to the half lines external to $G_{0}$, respecting cyclic ordering (e.g. keep fixed the two half lines, say $i_{2}$ and $i_{3}$ which contract outside $G_{0}$ if $i$ is lower than $j$ in the arbitrary order chosen). Repeating this algorithm, a unique $(G, \mathfrak{F})$ is obtained from an ordered $\left(G^{\prime}, \mathfrak{F}^{\prime}, \sigma\right)$ as in [24].

There remains only to count how many different ordered $\left(G^{\prime}, \mathfrak{F}^{\prime}, \sigma\right)$ are mapped on $(G, \mathfrak{F})$ by $\varphi$, hence by induction, count the possibilities for the reverse algorithm. With a factor $\frac{1}{2} k(j) \cdot[k(j)-1]$, one chooses a pair of numbers between 1 and $k(j)$; the lowest tells us where to insert $G_{0}$ in $G_{j}^{\prime}$ and the highest tells us which order should be given to the second (created) vertex of $G_{0}$ in the "arbitrary order" relative to $G_{j}^{\prime}$. Finally with a factor $n^{\prime}$ we choose which number one should give to the created vertex, and there are $16=4 \times 4$ ways of numbering the half lines of $G_{0}$ according to the reverse contraction process chosen above. Therefore there are by induction $\frac{n^{\prime} !}{n !} \prod_{j}\left\{k(j) !(k(j)-1) ! c^{k(j)-1}\right\}$ (with $c=8$ ) ordered contributions mapped by $\varphi$ on $(G, \mathfrak{F})$. Taking into account the weight $\prod_{j}[k(j) !]^{-1}$ in front of each of them, one gets Lemma A.2.

To prove (III.1), one puts together (II.12), (A.6), (A.8), and (A.11). The factor $\frac{n^{\prime} !}{n !}$ in (A.11) takes into account the corresponding factors $\frac{1}{n\left(G^{\prime}\right) !}$ and $\frac{1}{n(G) !}$. The factor $\prod_{j=1}^{n}[k(j)-1]$ ! in (A.11) cancels the corresponding one in (A.8), the factor $C\left(\mathfrak{F}_{j}^{\prime}\right)$ in (A.8) is taken into account by the counting of the sectors $\sigma$ in the triplets considered in Lemma A.1, and it remains only to verify that

$$
\prod_{j=1}^{n}\left\{\sum_{k(j)=1}^{\infty}\left[-g \cdot c \cdot \xi\left(\alpha_{j}\right)\right]^{k(j)-1}\right\}
$$

has the required properties for the factor $\mathrm{OD}_{G}^{\mathbb{\Im}}$ in Theorem III.1. But this is obvious since for $g$ sufficiently small, depending on $[\alpha], g \cdot c \cdot \xi\left(\alpha_{j}\right)$ is smaller than 1 and the series in (A.12) converge absolutely to $\prod_{j=1}^{n} \operatorname{od}\left(\alpha_{j}\right)$, with

$$
\operatorname{od}\left(\alpha_{j}\right) \equiv\left[1+g \cdot c \cdot \xi\left(\alpha_{j}\right)\right]^{-1}
$$

Note that (A.13) always makes sense if $g$ is not a negative real number, even when (A.12) is no more absolutely convergent. This is due to the crucial alternating signs 
in (A.12), which can be traced back to the minus signs in $\prod_{p=1}^{k(j)-1}\left(-t_{F_{j}^{p}}\right)$ in (A.6), and further back to the asymptotic freedom of the model.

The factors od $\left(\alpha_{v}^{*}\right)$ at each "ordinary" vertex $v$ go to zero when the $\alpha$ 's go to 0 (the ultra-violet region), because (A.7) contains a logarithmically divergent integral. This ultra-violet improvement of the dressed integrand in the right-hand side of (III.1) is important for the convergence theorems of the next appendix. The following elementary estimates will be used:

Lemma A.2. There exists a positive constant a such that:

$$
\begin{aligned}
& 0 \leqq c \xi(x) \leqq a \quad \text { for } x \geqq 1, \\
& c|\log x|-a \leqq c \xi(x) \leqq c|\log x|+a \quad \text { for } \quad 0<x \leqq 1 \text {. }
\end{aligned}
$$
Proof. (A.14) is trivial. For (A.15), one remarks that $\int_{0}^{1} \frac{2 d \beta}{(1+\beta)^{2}}=1$ and that for
$0<x \leqq 1,|| \log x|-\xi(x)|$ is bounded by

$$
\int_{1}^{\infty} \frac{d \alpha}{\alpha} e^{-\alpha}+\int_{0}^{1} \frac{d \alpha}{\alpha} \int_{0}^{1}\left[1-e^{-\alpha(1+\beta)}\right] \frac{2 d \beta}{(1+\beta)^{2}} .
$$

Now let us define precisely the projection $\psi$.

Definition A.3. A contribution $(G, \mathfrak{F},[\alpha])$ is "fundamental" if and only if it is intermediate and, for any $F \in \mathfrak{Q}(\mathfrak{F})$ with $F / \mathfrak{Q}(\mathfrak{F})$ isomorphic to $G_{0}, \mathfrak{A}_{\mathfrak{Q}(\mathfrak{F})}(F)$, defined by (II.10) has exactly two elements.

Remark that in the statement above, $F / \mathfrak{Q}(\mathbb{F})$ cannot have two ordinary vertices since $(G, \mathfrak{F},[\alpha])$ is intermediate. Therefore it could have 1 or 2 reduction vertices (respectively, $\mathfrak{A}_{\mathfrak{Q}(\mathfrak{Z})}(F)$ could have 1 or 2 elements). Definition A.3 means that for a fundamental contribution the first case is excluded.

One has the following analog of Proposition A.1:

Proposition A.2. An intermediate contribution $(G, \mathfrak{F},[\alpha])$ is fundamental if and only if there does not exist any subforest $\mathfrak{F}^{\prime} \subseteq \mathfrak{Q}(\mathfrak{F})$, totally ordered by inclusion, with at least two elements, such that if $F_{0}^{\prime}$ is its minimal element, $F / \mathfrak{F}^{\prime}$ is isomorphic to $G_{0}$ for any $F \in \mathfrak{F}, F \neq F_{0}^{\prime}$.

In other words there does not exist $\mathfrak{F}^{\prime} \subseteq \mathfrak{F}$, such that $\mathfrak{F}^{\prime}=\left\{F_{0}^{\prime}, \ldots, F_{k}^{\prime}\right\}, k \geqq 1$, $F_{0}^{\prime} \subset F_{1}^{\prime} \ldots \subset F_{k}^{\prime}, F_{0}^{\prime} \in \mathfrak{Q}(\mathfrak{F}), F_{j}^{\prime} / F_{j-1}^{\prime}$ is isomorphic to $G_{0}$ for $j=1, \ldots, k$ and:

$$
\alpha^{*}\left(F_{k}^{\prime}, \mathfrak{F}\right) \leqq \sup _{i \in F_{k}^{\prime} / F_{k-1}^{\prime}} \alpha_{i} \leqq \ldots \leqq \sup _{i \in F_{j}^{\prime} / F_{j}^{\prime}-1} \alpha_{i} \leqq \ldots \leqq \sup _{i \in F_{1}^{\prime} / F_{0}^{\prime}} \alpha_{i} \leqq \alpha\left(F_{0}^{\prime}, \mathfrak{F}\right)
$$

Proof. Again we play with the fact that here, for $1 \leqq j \leqq k$,

$$
\alpha\left(F_{j}^{\prime}, \mathfrak{F}\right)=\alpha^{*}\left(F_{j-1}^{\prime}, \mathfrak{F}\right)=\sup _{i \in F_{j}^{\prime} / F_{j-1}^{\prime}} \alpha_{i}
$$

$\psi$ is a projection from the set of intermediate contributions onto the fundamental ones. Let $\left(G^{\prime}, \mathfrak{F}^{\prime},\left[\alpha^{\prime}\right]\right)$ be an intermediate contribution. We identify the maximal subforests $\mathfrak{F}^{1}, \ldots, \mathfrak{F}^{k}$ of $\mathfrak{Q}(\mathfrak{F})$ which verify all the conditions of Proposition A.2. To obtain $\psi\left[\left(G^{\prime}, \mathfrak{F}^{\prime},\left[\alpha^{\prime}\right]\right)\right] \equiv(G, \mathfrak{F},[\alpha])$, we reduce every $F / \mathfrak{F}_{i}$, for $F \in \mathfrak{F}_{i}$, to a single reduction vertex, except the minimal subgraphs $F_{0}^{1}, \ldots, F_{0}^{k}$, which 


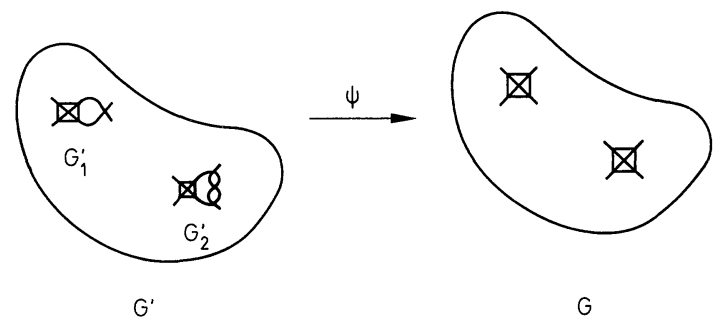

Fig. 3. The mapping $\psi$ on graphs

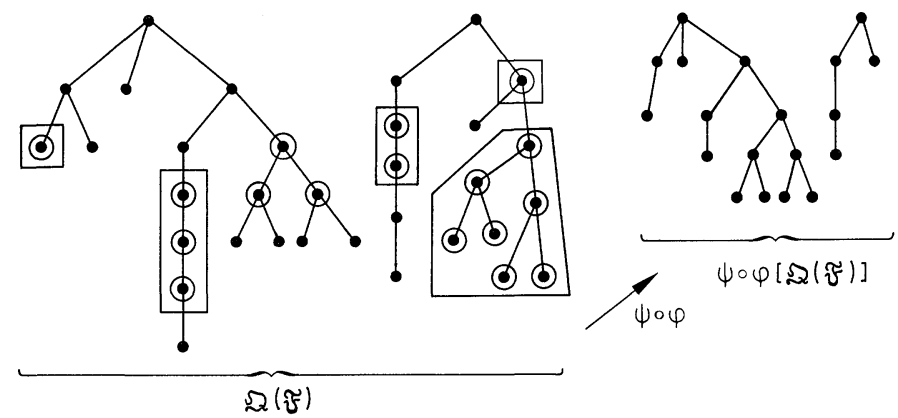

Fig. 4. The mapping $\psi \circ \varphi$ acting on $\mathfrak{Q}(\mathfrak{F})$

- $F \in \mathfrak{Q}(\mathfrak{F})$

$\odot \quad F / \mathfrak{Q}(\mathfrak{F}) \simeq G_{0}$

$\odot F$ contracted by $\psi \circ \varphi$

are kept unchanged. Like for $\varphi$, the forest $\mathfrak{F}$ and the set $[\alpha]$ are obtained from $\mathfrak{F}^{\prime}$ and $\left[\alpha^{\prime}\right]$ by suppressing the reduced subgraphs and their internal $\alpha$-parameters. This is sketched in Fig. 3. In Fig. 4, the projection $\psi \circ \varphi$ is sketched from the point of view of forests with their natural partial ordering, the inclusion. Maximal elements are on top of the figure, and connecting lines represent inclusion relations. The subgraphs $F$ in $\mathfrak{Q}(\mathfrak{F})$ with $F / \mathfrak{Q}(\mathfrak{F})$ isomorphic to $G_{0}$ are circled. The projection $\psi \circ \varphi$ reduces the maximal blocks of circled points at the bottom of the forest ( $\varphi$-reduction) plus all maximal linear chains of circled elements anywhere in the forest ( $\psi$-reduction), as shown in Fig. 4.

It is left to the reader to check that in the reduction described above there cannot appear in $(G, \mathfrak{F},[\alpha])$ new subgraphs $F \in \mathfrak{F}$ with $F / \mathfrak{Q}(\mathfrak{F})$ isomorphic to $G_{0}$ and having 0 or 1 reduction vertex.

Now to prove (III.2) we follow the proof of (III.1). Let us consider an intermediate $\left(G^{\prime}, \mathfrak{F}^{\prime},[\alpha]\right)$ with $\psi\left[\left(G^{\prime}, \mathfrak{F}^{\prime},\left[\alpha^{\prime}\right]\right)\right]=(\mathrm{G}, \mathfrak{F},[\alpha])$. Every quadruped $F_{j}^{0}, j=1, \ldots, s$ of $\mathfrak{Q}(\mathfrak{F})$ is the minimal element of a totally ordered subforest of $\mathfrak{F}^{\prime}, \mathfrak{F}_{j}^{\prime}=\left\{F_{j}^{0} \subset \ldots \subset F_{j}^{k(j)}\right\}$, which satisfy the conditions of Proposition A.2, except that $k(j)$ could be now 0 . For $1 \leqq p \leqq k(j)$, we call $\alpha_{j}^{p}$ the supremum of the $\alpha$-parameters of $F_{j}^{p} / F_{j}^{p-1}$, and $\alpha_{j}^{p} \beta_{j}^{p}$ the parameter of the other line of $F_{j}^{p} / F_{j}^{p-1}$. We define also $\alpha_{j} \equiv \alpha\left(F_{j}^{0}, \mathfrak{F}\right)$ and $\alpha_{j}^{*} \equiv \alpha^{*}\left(F_{j}^{0}, \mathfrak{F}\right)=\alpha^{*}\left(F_{j}^{k(j)}, F^{\prime}\right)$. Putting $n(G) \equiv n$ and 
$n\left(G^{\prime}\right) \equiv n^{\prime}$, the integration in (III.2) can be written as:

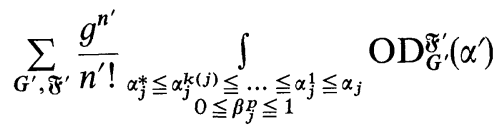

$$
\begin{aligned}
& \ldots \prod_{j=1}^{s}\left\{\prod_{p=1}^{k(j)}\left[2 \alpha_{j}^{p} d \alpha_{j}^{p} d \beta_{j}^{p} \exp \left[-\alpha_{j}^{p}\left(1+\beta_{j}^{p}\right)\right]\right] T_{\widetilde{\mho}_{J}^{\prime}}\right\} T_{\widetilde{F}^{\prime}} Z_{G^{\prime}}\left(\mathbf{p}, \alpha^{\prime}\right) .
\end{aligned}
$$

Like in (A.6) the $T_{\widetilde{\gamma}_{j}^{\prime}}$ operators cut the integrand $Z_{G^{\prime}}$ into

$$
Z_{G} \prod_{j=1}^{p} \prod_{p=1}^{k(j)}\left[\alpha_{j}^{p}\left(1+\beta_{j}^{p}\right)\right]^{-2}
$$

Moreover every $F_{j}^{p} / \mathfrak{F}^{\prime}$ has exactly one ordinary vertex and one reduction vertex. Therefore, using (A.13):

$$
g^{n^{\prime}-n} \mathrm{OD}_{G^{\prime}}^{\widetilde{F}^{\prime}}\left(\alpha^{\prime}\right)=\mathrm{OD}_{G}^{\widetilde{\gamma}}(\alpha) \prod_{j=1}^{s} \prod_{p=1}^{k(j)}\left[\frac{g}{1+g \cdot c \cdot \xi\left(\alpha_{j}^{p}\right)}\right] .
$$

Let us define, for $0 \leqq x \leqq y$, the function

$$
\zeta(x, y) \equiv \int_{x}^{y} \frac{d \alpha}{\alpha}\left[\frac{g}{1+g \cdot c \cdot \xi(\alpha)}\right] \int_{0}^{1} \exp [-\alpha(1+\beta)] \frac{2 d \beta}{(1+\beta)^{2}} .
$$

It is easy to perform the integration in (A.17), obtaining:

$$
g^{n}\left\{\prod_{j=1}^{s} \frac{1}{k(j) !}\left[-\zeta\left(\alpha_{j}^{*}, \alpha_{j}\right)\right]^{k(j)}\right\} \operatorname{OD}_{G}^{\widetilde{F}}(\alpha) T_{\widetilde{F}} Z_{G}(\mathbf{p}, \alpha) .
$$

The following combinatoric lemma allows us to perform exactly the summation of all intermediate contributions which dress a fundamental one.

Lemma A.3. Let $(G, \mathfrak{F},[\alpha])$ be a fundamental contribution with $\mathfrak{Q}(\mathfrak{F})=\left\{F_{1}^{0}, \ldots, F_{s}^{0}\right\}$. The number of pairs $\left(G^{\prime}, \mathfrak{F}^{\prime}\right)$ obtained from $G$ and $\mathfrak{F}$ by the insertion of the forests $\mathfrak{F}_{1}^{\prime}, \ldots, \mathfrak{F}_{s}^{\prime}$ considered in Proposition (A.2) is

$$
\frac{n^{\prime} !}{n !} \prod_{j=1}^{s}[2 c]^{k(j)}
$$

Proof. We follow Lemma A.1. There are $\frac{n^{\prime} !}{n !}$ ways of ordering the $n^{\prime}$ vertices of $G^{\prime}$ which respect the relative ordering of the vertices of $G$. One proves (A.21) by induction, in the same way as Lemma A.1. Instead of choosing a pair of indices (hence the factor $k(j) \cdot \frac{k(j)-1}{2}$ in the proof of Lemma A.1) one chooses only the index of the ordinary vertex of $G_{0}$; then there is a factor 16 to insert this $G_{0}$, respecting all cyclic orderings. This proves (A.21) because $16=2 \times 8=2 c$. The reader is urged to verify this numerical value since the factors $2^{k(j)}$ in (A.21) will be important for the rest of the paper, Lemma A.4 and Appendix B below. This factor 2 corresponds intuitively to the breaking of the symmetry of $G_{0}$ by imposing the existence of one particular vertex, the reduction vertex. 
Putting together (A.19) and Lemma A.3 achieves the proof of (III.2), hence of Theorem (III.1), with:

$$
D_{G}^{\widetilde{\mho}}(\alpha)=\mathrm{OD}_{G}^{\widetilde{\gamma}}(\alpha) \cdot \prod_{j=1}^{s} \exp \left[-2 c \cdot \zeta\left(\alpha_{j}^{*}, \alpha_{j}\right)\right],
$$

which has the desired structure (III.3)-(III.5) if one defines

$$
\operatorname{rd}(x, y) \equiv \exp [-2 c \cdot \zeta(y, x)]
$$

The condition $\alpha^{*}\left(F_{j}^{0}, \mathfrak{F}\right) \leqq \alpha\left(F_{j}^{0}, \mathfrak{F}\right)$, or $\alpha_{j}^{*} \leqq \alpha_{j}$, which is required for (A.22) and (A.23) to make sense [see (A.19)], is precisely $F_{j}^{0} \in \mathfrak{Q}(\mathfrak{F})$. Again the formalism of "skeleton forests" developed in [1] appears to be an adequate tool.

What has been gained in this second dressing operation is crucial. First, in contrast with $\operatorname{OD}_{G}^{\mathbb{F}}(\alpha)$, the dressing factors $D_{G}^{\mathbb{\mho}}(\alpha)$ decrease when the $\alpha$-parameters attached to reduction vertices of $\mathfrak{Q}(\mathfrak{F})$ tend to 0 , allowing us to transport convergence from inside graphs in $\mathfrak{Q}(\mathfrak{F})$ to the outside. Second, the mapping $\psi$ "trims" some of the remaining parquet structures in intermediate contributions (see the remark after Lemma B.2).

The following elementary estimates will be used in the next appendix and show how $D_{G}^{\widetilde{F}}(\alpha)$ decreases when the ratios $\alpha_{F}^{*} / \alpha_{F}$ become small.

Lemma A.4. There exists a positive constant $b$ such that for $\operatorname{Re} g>0,|g|<\varepsilon$ and $\varepsilon$ small enough, one has:

$$
\begin{array}{ll}
\text { for } & 1 \leqq x \leqq y|\exp -2 c \cdot \zeta(x, y)| \leqq 1 \\
\text { for } \quad 0<x \leqq 1 \leqq y|\exp -2 c \cdot \zeta(x, y)| \leqq \frac{b}{[1+|g| \cdot c \cdot|\log x|]^{2}} & \\
\text { for } \quad 0<x \leqq y \leqq 1|\exp -2 c \zeta(x, y)| \leqq b\left[\frac{1+|g| \cdot c \cdot|\log y|}{1+|g| \cdot c \cdot|\log x|}\right]^{2} .
\end{array}
$$

Proof. Remark that $|\exp -2 c \zeta| \leqq[\exp -c \cdot \operatorname{Re} \zeta]^{2}$. By (A.14), $c \xi(\alpha) \geqq 0$ if $\alpha \geqq 1$, hence $\operatorname{Re} \frac{g}{1+g \cdot c \cdot \xi(\alpha)}>0$ for $\operatorname{Re} g>0$. This implies $\operatorname{Re} \zeta(x, y)>0$ for $1 \leqq x \leqq y$, hence (A.24).

Putting $\lambda=1 / g \cdot c,(\mathrm{~A} .25)$ is implied by:

$$
\int_{x}^{1} \frac{d \alpha}{\alpha} \operatorname{Re} \frac{1}{\lambda+\xi(\alpha)} \int_{0}^{1} e^{-\alpha(1+\beta)} \frac{2 d \beta}{(1+\beta)^{2}} \geqq \log \left[\frac{|\lambda|+|\log x|}{|\lambda|}\right]-K,
$$

where $K$ is a constant (independent of $x$ and of $\lambda$ ), provided $\operatorname{Re} \lambda>0$ and $|\lambda|<\frac{1}{\varepsilon \cdot c}$. Indeed (A.25) follows from (A.27) with $b=\exp (2 K c)$. We write $\log \left[\frac{|\lambda|+|\log x|}{|\lambda|}\right]$ $=\int_{0}^{|\log x|} \frac{d t}{|\lambda|+t}$; with $t=|\log x|$, we subtract this from (A.27), which becomes a 
consequence of the stronger assertion:

$$
\begin{gathered}
\int_{0}^{|\log x|} d t\left|\operatorname{Re}\left(\frac{1}{\lambda+\xi\left(e^{-t}\right)}-\frac{1}{\lambda+t}\right)\right|+\left|\operatorname{Re}\left(\frac{1}{\lambda+t}\right)-\frac{1}{|\lambda|+t}\right| \int_{0}^{1} e^{-e^{-t}(1+\beta)} \frac{2 d \beta}{(1+\beta)^{2}} \\
\ldots+\int_{x}^{1} \frac{d \alpha}{\alpha} \frac{1}{|\lambda|+|\log \alpha|} \int_{0}^{1}\left[1-e^{-\alpha(1+\beta)}\right] \frac{2 d \beta}{(1+\beta)^{2}} \leqq K
\end{gathered}
$$

But

$$
\int_{x}^{1} \frac{d \alpha}{\alpha} \frac{1}{|\lambda|+|\log \alpha|} \int_{0}^{1}\left[1-e^{-\alpha(1+\beta)}\right] \frac{2 d \beta}{(1+\beta)^{2}} \leqq \varepsilon \cdot c \int_{0}^{1} \int_{0}^{1} \frac{2 d \alpha d \beta}{1+\beta}
$$

is a bounded integral. Moreover one has, for some $\lambda$-independent $K^{\prime}$ :

$$
\int_{0}^{\infty} d t\left|\operatorname{Re}\left(\frac{1}{\lambda+\xi\left(e^{-t}\right)}-\frac{1}{\lambda+t}\right)\right|+\left|\operatorname{Re}\left(\frac{1}{\lambda+t}\right)-\frac{1}{|\lambda|+t}\right| \leqq K^{\prime} .
$$

Indeed, using $\left|\operatorname{Re} \frac{u}{v}\right| \leqq \frac{|u|}{|v|}$ and $\left|\xi\left(e^{-t}\right)-t\right| \leqq a$, (A.15), it suffices to show:

$$
\int_{0}^{\infty} d t\left|\frac{a}{\left(\lambda+\xi\left(e^{-t}\right)\right)(\lambda+t)}\right|+\left|\frac{2 \lambda}{(\lambda+t)(|\lambda|+t)}\right| \leqq K^{\prime} .
$$

Choosing $\varepsilon<\frac{1}{2 c \cdot a}$, using (A.15) and $\operatorname{Re} \lambda>0$ gives

$$
\left|\lambda+\xi\left(e^{-t}\right)\right| \geqq \sup \left(\frac{|\lambda|}{2}, t\right) \geqq \frac{|\lambda|+t}{4},
$$

and $|\lambda+t| \geqq \frac{|\lambda|+t}{2}$, and the left-hand side of (A.30) is bounded by $\int_{0}^{\infty} \frac{8 a+4|\lambda|}{[|\lambda|+t]^{2}} d t \leqq 8$, using the condition $\varepsilon<\frac{1}{2 c \cdot a} \Rightarrow 2 a<|\lambda|$.

This achieves the proof of (A.28), hence of (A.25). The proof of (A.26) is similar.

\section{Appendix B: Convergence of the Dressed Expansion}

In this appendix we prove Theorem III.2 which states that the sum over dressed fundamental contributions performed in a well defined way is an absolutely convergent one. Note that this "sum" includes integrations over $\alpha$-parameters.

For $g$ complex with $\operatorname{Re} g>0,|g|<\varepsilon$, we have

$$
\left|g[1+g \cdot c \cdot \xi(\alpha)]^{-1}\right| \leqq \inf \left\{\varepsilon,[c \cdot \xi(\alpha)]^{-1}\right\} .
$$

We give our convergence proof only for $g$ real and positive, $g<\varepsilon$. The extension of the arguments to $g$ complex with $\operatorname{Re} g>0$ and $|g|<\varepsilon$ is easy, since the bounds (B.1) and (A.24)-(A.26), which are uniform in this domain, are the only ones we use. The convergence being uniform, the analyticity in $g$ of the sum $S_{e}(\mathbf{p}, g)$ is an easy consequence of the analyticity in $g$ of the factors $D_{G}^{\mathbb{F}}(\alpha)$. 
We do not need to count Wick contractions any more, therefore we return to unlabeled graphs. Let $K_{G}^{\mathfrak{F}, \mathfrak{H}}(\mathbf{p}) \equiv n ! J_{G}^{\mathfrak{F}, \mathfrak{H}}(\mathbf{p})$. From (III.7) and (III.2), one has

$$
\begin{gathered}
K_{G}^{\mathfrak{F}, \mathfrak{H}}(\mathbf{p})=g^{n} \int_{\Delta \widetilde{\mho}, \mathfrak{H}} d \mu(\alpha) D_{G}^{\mathfrak{F}}(\alpha) \prod_{H \in \mathfrak{H}}\left(1-\mathbf{t}_{H}\right) Z_{\mathfrak{G}}^{\mathfrak{F}}(\mathbf{p}, \alpha), \\
Z_{\mathfrak{G}}^{\mathfrak{F}}(\mathbf{p}, \alpha) \equiv T_{\mathfrak{F}} Z_{G}(\mathbf{p}, \alpha) .
\end{gathered}
$$

We say that $K_{G}^{\mathfrak{F}, \mathfrak{H}}$ is "a fundamental piece" of the dressed expansion if $\Delta_{\mathfrak{F}, \mathfrak{H}} \neq \emptyset$, which will be now assumed. Theorem (III.2) follows from

Lemma B.1. There exists $K(\varepsilon)$ with $\lim _{\varepsilon \rightarrow 0} K(\varepsilon)=0$, such that for $0<g<\varepsilon$ :

$$
\left|K_{G}^{\mathfrak{F}, \mathfrak{S}}(\mathbf{p})\right| \leqq[K(\varepsilon)]^{n(G)} .
$$

Proof of Theorem III.2. Assuming Lemma B.1, one gets rid of the factor $n(G)$ ! in (II.12) and performs the sum in (III.8) over unlabeled graphs with $J_{G}^{\mathfrak{F}, \mathfrak{H}}$ replaced by $K_{G}^{\mathbb{F}, \mathfrak{H}}$. The number of planar graphs (unlabeled) at order $n$ is bounded by (const) ${ }^{n}$ [39-40], and the number of closed divergent forests in a graph with $n$ vertices is bounded by $8^{n}$ [1, Lemma A.2]. Therefore we can fix $\varepsilon$ small enough so that the series in (III.8) is dominated uniformly by a convergent geometric series in $n(G)$.

Remark. (B.4), which is the key estimate of this paper will be proved now for general graphs, planar or not (see the end of Sect. IV).

Proof of Lemma B.1. 1. Case with No Bideps. Bideps create technicalities; therefore we prove first Lemma B.1 for a graph $G$ without bipeds, hence one has always $S(\mathfrak{F})$ $=\mathfrak{Q}(\mathfrak{F})$. The general case is considered at the end of the appendix. From now on, a fundamental piece $K_{G}^{\mathbb{F}, \mathfrak{H}}$ is fixed.

We write $|\mathfrak{F}|$ for the number of elements in the forest $\mathfrak{F}$. By [1, Lemma C.1],

$$
|\mathfrak{F} \cup \mathfrak{H}|=|\mathfrak{F}|+|\mathfrak{H}|<n(G) .
$$

Our main problem is to evaluate the integral (B.2) when $G$ contains large parquet pieces, so that $|\mathfrak{F} \cup \mathfrak{S}| \simeq n(G) \equiv n$. In this case, we cannot use only the logarithmic factors in $D_{G}^{\mathscr{F}}$ to bound the UV integrations, without performing any $\left(1-\mathbf{t}_{H}\right)$ renormalization in (B.2). Indeed this would eat one logarithm, hence by (B.1) one factor $g$ per divergent integration. The total number of these integrations, $|\mathfrak{F} \cup \mathfrak{H}|$ being close to $n$, one would not have enough remaining $g$ 's to ensure the decrease of $K(\varepsilon)$ in (B.4) as $\varepsilon \rightarrow 0$. On the other hand if one performs $h$ subtractions $\left(1-t_{H}\right)$ in (B.2) with $h$ large ( $h / n$ bounded below by $\eta>0$ for instance), a disastrous $h$ ! appears in the $\alpha$-representation [1, Lemma III.4] which we do not know how to eliminate. Our solution combines renormalization of carefully chosen subgraphs $H$ and use of the logarithmic factors in $D_{G}^{\widetilde{F}}$ for the others. There exists indeed a mixed $\alpha$ - and momentum representation (B.13) which does not contain factorial terms for disjoint renormalized subgraphs with a bounded number of vertices. Fixing this number to be large, one can bound the integrations over the remaining divergent subgraphs, which are then very large, by using the "accumulated" logarithms in $D_{G}^{\tilde{S}}$, and get (B.4). 
The whole method works because in contrast with $\frac{|\mathfrak{F} \cup \mathfrak{H}|}{n}, \frac{|\mathfrak{F}|}{n}$ is bounded away from 1:

\section{Lemma B.2.}

$$
|\mathfrak{F}| \leqq \frac{2 n}{3}
$$

Proof. Let $\Omega$ be the subforest of $\mathfrak{F}$ made of these $F \in \mathfrak{F}$ which verify $F / \mathfrak{F}$ isomorphic to $G_{0}$. For any such $F \in \mathfrak{R}, \mathfrak{U}_{\mathfrak{F}}(F)=2$ by Definition A.3. This implies $|\mathfrak{R}| \leqq \frac{|\mathfrak{F}|}{2}$, which implies (B.6) by an argument similar to [1, Lemma C.1].

Remark. There is no analog of Lemma B.2 for intermediate contributions. Here again the necessity of the second dressing $\psi$ appears.

Let $q$ be an integer which will be fixed later to a large value independent of $G, \mathfrak{F}$, $\mathfrak{H}$, and $n$. We define $\mathfrak{I}$ and $\mathfrak{I}$ by:

$$
\begin{gathered}
\mathfrak{I} \equiv\{H \in \mathfrak{H} / n(H / \mathfrak{F}) \leqq q\}, \\
\mathfrak{I} \equiv\{H \in \mathfrak{H} / n(H / \mathfrak{F}) \geqq q+1\},
\end{gathered}
$$

and $\mathfrak{L}=\mathfrak{F} \cup \mathfrak{I}$. One has

$$
\begin{aligned}
& K_{G}^{\mathfrak{G}, \mathfrak{H}}=\sum_{\mathscr{\mho} \leqq \mathfrak{R} \cong \mathfrak{L}} K_{G}^{\mathfrak{F}, \mathfrak{H}, \mathfrak{A}}, \\
& K_{G}^{\mathfrak{F}, \mathfrak{H}, \boldsymbol{\Re}} \equiv g^{n} \int_{\Delta \mathfrak{F}, \mathfrak{H}} d \mu(\alpha) D_{G}^{\mathscr{F}}(\alpha) \prod_{H \in \mathfrak{J}}\left(1-t_{H}\right) Z_{G}^{\mathfrak{S}}(\mathbf{p}, \alpha) .
\end{aligned}
$$

Let us define also $\mathfrak{I}_{m} \equiv\left\{H \in \mathfrak{I}, H \neq G / B_{\mathfrak{I} \cup \mathfrak{Y}}(H) \in \mathfrak{F} \cup\{G\}\right\}$. $\mathfrak{I}_{m}$ consists of the maximal elements of $\mathfrak{I}$ in the various $F \in \mathfrak{F} \cup\{G\}$. For $F_{1} \in \mathfrak{I}_{m}$ and $F_{2} \in \mathfrak{I}_{m}$, one has $F_{1} / \mathfrak{R}=F_{1} / \mathfrak{F}$ and $F_{2} / \mathfrak{R}=F_{2} / \mathfrak{F}$, and $F_{1} / \mathfrak{F}$ and $F_{2} / \mathfrak{F}$ are disjoint. This property allows us to write representation (B.13) below.

Furthermore we define for $H \in \mathfrak{I}_{m}, \mathfrak{F}(H) \equiv\left\{F \in \mathfrak{F}\right.$, with $\left.B_{\mathfrak{F} \cup\{H\}}(F)=H\right\}$, the forest of the "reduction vertices" of $H$, and

$$
\mathfrak{H}(H)=\left\{H^{\prime} \in \mathfrak{H} / B_{\mathfrak{F} \cup\{H\}}\left(H^{\prime}\right)=H\right\} \cup\{H\},
$$

which is a subforest of $\mathfrak{I}$ by (B.7) and (B.8). One has $\mathfrak{I}=\bigcup_{H \in \mathfrak{I}_{m}} \mathfrak{H}(H)$.

Finally for $F \in \mathfrak{F}$ we define:

$$
\begin{aligned}
& F \in \mathfrak{F}_{1} \Leftrightarrow \alpha_{F} \geqq \alpha_{B_{\mathfrak{I}}(F)}, \\
& F \in \mathfrak{J}_{1} \Leftrightarrow \alpha_{F} \leqq \alpha_{B_{\mathfrak{Q}}(F)} .
\end{aligned}
$$

Remark that $\mathfrak{F}_{1} \subseteq \mathfrak{F}, \mathfrak{I}_{1} \subseteq \mathfrak{F}, \mathfrak{F}_{1} \cap \mathfrak{I}_{1}=\emptyset$, and $\mathfrak{L}=\mathfrak{F}_{1} \cup \mathfrak{I}_{1} \cup \mathfrak{J}$.

We will use the following mixed representation:

\section{Lemma B.3.}

$$
\begin{aligned}
& K_{\boldsymbol{G}}^{\mathfrak{F}, \mathfrak{H}, \mathcal{I}}(\mathbf{p})=g^{n} \int_{\Delta \widetilde{F}, \mathfrak{G}} d \mu(\alpha) D_{G}^{\mathfrak{F}}(\alpha) \int \prod_{H \in \mathfrak{\Im}_{m}}\left[Z_{H / \mathfrak{F}}^{R}\left(\mathbf{p}_{H}\right)\right]
\end{aligned}
$$

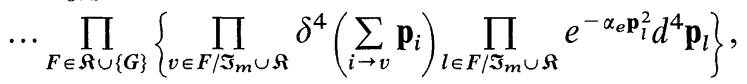


where in (B.13) there is a 4-momentum $\mathbf{p}_{l}$ for each internal line of $F / \mathfrak{I}_{\mathfrak{m}} \cup \mathfrak{R}$, $F \in \mathfrak{R} \cup\{G\}$; a momentum conservation law $\delta^{4}\left(\sum_{i \rightarrow v} \mathbf{p}_{i}\right)$ at each vertex $v$ of $F / \mathfrak{I}_{m} \cup \mathfrak{H}$, and $Z_{H / \widetilde{P}}^{R}$ is defined by:

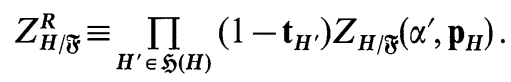

In (B.14) the $\alpha$-parameters of the internal lines of $H / \mathfrak{F}$ are called $\alpha^{\prime}$, and $\mathbf{p}_{H}$ is the set of the 4 external momenta of $H / \mathfrak{F}$, hence the momenta incident to the "reduction vertex" corresponding to $H$ in (B.13).

Proof. Lemma B.3 is a straightforward consequence of the way the renormalization acts in momentum space [47], and of the equivalence of the momentum and $\alpha$-renormalization $([42,43])$.

Now we want to apply (A.14) and (A.15) and (A.24)-(A.26). We cut the domain of $\alpha$-integrations into at most $2^{l(G)} \leqq 4^{n}$ pieces, according to which $\alpha$ 's are smaller than 1 . Actually the integration in the infra-red regions $1 \leqq \alpha<\infty$ are easier, and we will consider only the most difficult region where all $\alpha$ 's lie between 0 and 1 (see, however, [61] for a correct treatment of infra-red integrations according to the method of [1]).

Applying (A.15) and (A.26) one gets, for $\varepsilon<\frac{a}{2}$ :

$$
D_{G}^{\mathscr{\Im}}(\alpha) \leqq D_{G}^{\mathscr{F}}(\alpha) \equiv \prod_{v \in G} \frac{1}{1+g \cdot c \cdot\left|\log \alpha_{v}^{*}\right|} \prod_{F \in \mathscr{F}}\left[\frac{1+g \cdot c \cdot\left|\log \alpha_{F}\right|}{1+g \cdot c \cdot\left|\log \alpha_{F}^{*}\right|}\right]^{2},
$$

where $A \leqq{ }^{n} B$ means $A \leqq K^{n} B$ for a fixed constant $K$.

We want to integrate first in (B.13) over the internal $\alpha^{\prime}$-parameters of $H / \mathfrak{F}$ for any $H \in \mathfrak{I}_{m}$. From now on, we call $\alpha$ the other parameters, and $\Delta(\alpha)$ the domain of all $\alpha^{\prime}$ with $\left[\alpha^{\prime}, \alpha\right] \in \Delta_{\mathfrak{F}, \mathfrak{H}}$.

Using the definitions of Sect. II.6, one shows easily that for any ordinary vertex $v$ of $G, \alpha_{v}^{*} \leqq \alpha_{B_{\mathfrak{Q}}(v)}$. Also for any $F \in \mathfrak{F}, \alpha_{F}^{*} \leqq \alpha_{B_{\mathfrak{Q}}(F)}$.

Therefore, using (B.11)-(B.12) and (B.15):

$$
D_{G}^{\widetilde{F}}(\alpha) \leqq E_{G}^{\mathfrak{F}}(\alpha) \equiv \prod_{v \in G} \frac{1}{1+g \cdot c \cdot\left|\log \alpha_{B(v)}\right|} \prod_{F \in \widetilde{F}_{1}}\left[\frac{1+g \cdot c \cdot\left|\log \alpha_{F}\right|}{1+g \cdot c \cdot\left|\log \alpha_{B(F)}\right|}\right]^{2},
$$

where we note $B(v)$ and $B(F)$ the subgraphs $B_{\mathfrak{Q}}(v)$ and $B_{\mathfrak{Q}}(F)$.

The important remark is that since $\mathfrak{I} \subseteq \mathfrak{H}, E_{\mathfrak{G}}^{\widetilde{F}}$ depends only on the parameters $\alpha$ and no more on any $\alpha^{\prime}$. Therefore we can integrate in (B.13) over the $\alpha^{\prime}$ parameters, using the following crude bound:

Lemma B.4.

$$
\int_{\alpha^{\prime} \in \Delta(\alpha)} d \mu\left(\alpha^{\prime}\right) Z_{H / \mathfrak{F}}^{R}\left(\alpha^{\prime}, \mathbf{p}_{H}\right) \leqq K^{n(H / \mathfrak{F})} \sum_{v=1}^{|\mathfrak{S}(H)|}(|\mathfrak{H}(H)|-v) !\left[\alpha_{H}^{*}\left|\mathbf{p}_{H}\right|\right]^{v},
$$

where $\left|\mathbf{p}_{H}\right|=1+4 \sum_{i=1}^{4}\left(\mathbf{p}_{H}^{i}\right)^{2}$ is defined as in [1], Eq. (II.6), and $K$ is a numerical constant. 
Proof. It is similar to the proof of Theorem 1 in [1], but uses the improved version of [1, Lemma III.4] introduced in [61] and the following comments. Since $\alpha^{\prime} \in \Delta(\alpha)$ we have $\alpha_{H^{\prime}} \leqq \alpha_{H}^{*}$ for any $H^{\prime} \in \mathfrak{S}(H)$. Therefore the left-hand side of (B.17) is just a piece of the renormalized amplitude $I_{H / \xi}^{R}$ according to the splitting (II.20) which was used in [1], with an overall infrared cutoff $\alpha_{H}^{*}$ on the parameters $\alpha$ of $H / \mathfrak{F}$. Therefore it can be estimated by a straightforward use of [1, Lemma III.4] and of [61]. $|\mathfrak{H}(H)|$ will be the analog of what is called $f(\mathfrak{F} \cup \mathfrak{H})$ in [1] and [61]. (In our case the analog of $s$ in [1, Lemma III.4], is just 1 , and $v$ is the analog of what is called $v$ in [61].) One gets, after integration on every Hepp's variable except one, the bound:

$$
\int_{\alpha^{\prime} \in \Delta(\alpha)} d \mu\left(\alpha^{\prime}\right) Z_{H / \widetilde{F}}^{R}\left(\alpha^{\prime}, \mathbf{p}_{H}\right) \leqq K^{n(H / \mathfrak{F})} \sum_{v=1}^{|\mathfrak{S}(H)|}(|\mathfrak{S}(H)|-v) !\left|\mathbf{p}_{H}\right|^{v^{\alpha_{H}^{*}}} \int_{0}^{*} \beta^{v-1} e^{-\beta} d \beta,
$$

where $\beta$ is the analog of what is called $\beta_{l}$ in [1]. Using $\int_{0}^{\alpha_{H}^{*}} \beta^{v-1} e^{-\beta} d \beta \leqq\left[\alpha_{H}^{*}\right]^{v}$ proves (B.17).

Using (B.16) and (B.17) we get from (B.13), (B.7) and the fact that $|\mathfrak{S}(H)| \leqq n(H / \mathfrak{F}) \leqq q:$

$$
\begin{aligned}
& \left|K_{G}^{\mathfrak{G}, \mathfrak{S}, \Omega}\right| \stackrel{n}{\leqq} g^{n} \int_{A} d \mu(\alpha)[q !]^{\left|\mathfrak{I}_{m}\right|} E_{G}^{\mathfrak{G}}(\alpha) \\
& \iint \ldots \prod_{H \in \mathfrak{I}_{m}}\left[\alpha_{H}^{*}\left|\mathbf{p}_{H}\right|\right]^{q} \prod_{F \in \mathcal{S} \cup\{G\}}\left\{\prod_{v \in F / \mathcal{S} \cup \mathfrak{I}_{m}} \delta^{4}\left(\sum_{i \rightarrow v} \mathbf{p}_{i}\right) \prod_{l \in F / \mathcal{S} \cup \mathfrak{I}_{m}} e^{-\alpha_{l} \mathbf{p}_{l}^{2}} d^{4} \mathbf{p}_{l}\right\},
\end{aligned}
$$

where $\Delta$ is the domain of all $\alpha$ 's which respect the collection of inequalities defining $\Delta_{\widetilde{8}, \mathfrak{F} .}$

Now we can get rid of the momentum representation, which was only technically useful in displaying the factorization (B.13) over disjoint subgraphs of $\mathfrak{I}_{m}$ without paying any factorial factor.

Let us consider $H \in \mathfrak{I}_{m}$, and let us order the parameters of its external lines as $\alpha_{1} \leqq \alpha_{2} \leqq \alpha_{3} \leqq \alpha_{4}$. We have $\alpha_{H}^{*}=\alpha_{2}$. By momentum conservation, $\left(\mathbf{p}_{H}^{1}\right)^{2} \leqq 3 \sum_{i=2}^{4}\left(\mathbf{p}_{H}^{i}\right)^{2}$. Therefore:

$$
\left[\alpha_{H}^{*}\left|\mathbf{p}_{H}\right|\right] \leqq \alpha_{H}^{*}+16 \alpha_{2} \sum_{i=2}^{4}\left(\mathbf{p}_{H}^{i}\right)^{2} \leqq \alpha_{H}^{*}+16 \sum_{i=2}^{4} \alpha_{i}\left(\mathbf{p}_{H}^{i}\right)^{2},
$$

which implies:

$$
\left.\delta^{4}\left(\sum_{i=1}^{4} \mathbf{p}_{H}^{i}\right)\left[\prod_{i=2}^{4} e^{-\alpha_{i}\left(\mathbf{p}_{H}^{i}\right)^{2}}\right]\left[\alpha_{H}^{*} \mid \mathbf{p}_{H}\right]\right]^{q} \leqq K(q) \delta^{4}\left(\sum_{i=1}^{4} \mathbf{p}_{H}^{i}\right) \prod_{i=1}^{4}\left[e^{-\frac{\alpha_{i}}{2}\left(\mathbf{p}_{H}^{i}\right)^{2}}\right]\left[1+\alpha_{H}^{*}\right]
$$

for some positive $K(q)$. Let $K^{\prime}(q)=q ! K(q)$. We make the change of variables $\alpha \rightarrow \alpha / 4$ (since a given line could be incident to two different graphs of $\mathfrak{I}_{m}$ ). Using (B.18) and (B.20) we get:

\section{Lemma B.5.}

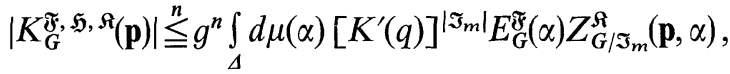

$$
\begin{aligned}
& Z_{G / \mathfrak{\Im}_{m}}^{\mathcal{S}}(\mathbf{p}, \alpha) \equiv \prod_{F \in \mathcal{S} \cup\{G\}} Z_{F / \mathfrak{\Im}_{m} \cup \mathcal{M}}(\mathbf{p}, \alpha) .
\end{aligned}
$$


Proof. The domain $\Delta$ is defined by a collection of inequalities between $\alpha$ 's and is therefore invariant by $\alpha \rightarrow \alpha / 4$. We have

$$
d \mu(\alpha)=\prod_{i} e^{-\alpha_{z}} d \alpha_{i} \leqq 4^{l^{\prime}} \prod_{i} e^{-\alpha_{i}} \frac{d \alpha_{i}}{4} \leqq \prod_{i} e^{-\frac{3}{4} \alpha_{i}} d \mu\left(\frac{\alpha_{i}}{4}\right),
$$

where $l^{\prime}=\sum_{F \in \mathcal{S} \cup\{G\}} l\left(F / \mathfrak{I}_{m} \cup \mathfrak{R}\right) \leqq 2 n$. Using (B.20) one performs the momentum integration and reconstructs $Z_{G / \mathfrak{I}_{m}}^{\mathcal{R}}(\mathbf{p}, \alpha)$, using the obvious bound $E_{G}^{\mathfrak{F}}(4 \alpha) \stackrel{n}{\leqq} E_{G}^{\mathfrak{F}}(\alpha)$ for $g$ small enough, and

$$
\left(1+\alpha_{H}^{*}\right) e^{-\frac{3}{4} \alpha_{H}^{*}} \leqq 2 .
$$

The important gain from (B.2) to (B.21) is that the renormalized divergent graphs in (B.21) are in $\mathfrak{S}$ instead of $\mathfrak{H}$, hence have at least $q+1$ generalized vertices, and the corresponding logarithms have been stored into $E_{G}^{\mathfrak{F}}$ without paying any factorial factor.

To simplify further, we use:

$$
Z_{G / \Im_{m}}^{\mathcal{R}}(\mathbf{p}, \alpha) \leqq Z_{G / \mathfrak{I}_{m}}^{\mathfrak{R}}(0, \alpha) \leqq Z_{G / \mathfrak{I}_{m}}^{\mathfrak{Q}}(0, \alpha) \equiv\left[U_{G / \mathfrak{I}_{m}}^{\mathfrak{R}}(\alpha)\right]^{-2} .
$$

The basic object we have to consider is the set $G / \mathfrak{I}_{m}$ made of $l^{\prime}$ lines which is the disjoint collection of the subgraphs $F / \mathfrak{I}_{m} \cup \mathfrak{L}, F \in \mathfrak{L} \cup\{G\}$ together with their partial ordering due to inclusion relations, which defines $B(F)$ for any $F \in \mathfrak{L}$. Let us call $n_{F}, l_{F}$ the number of vertices and lines of $F / \mathfrak{I}_{m} \cup \mathfrak{Q}$ which will be noted $F$ for short when no ambiguity is possible.

Let us define $\Delta_{\mathfrak{F}_{1}, X}$ as the subdomain of $\Delta$ which corresponds to a fixed $\mathfrak{F}_{1}$ in (B.11), $\mathfrak{F}_{1} \subseteq \mathfrak{F}$, and to a fixed set $X$ of lines with parameters $\alpha_{F}, F \in \mathfrak{L} \cup\{G\}$. Hence $|X|=|\mathfrak{L} \cup\{G\}|$. We will bound uniformly (B.18), where the integration is restricted to $\Delta_{\mathscr{F}_{1}, X}$ and prove therefore Lemma B.1, since there is at most $8^{n} \cdot 2^{l^{\prime}} \leqq 1$ choices for $\left(\mathfrak{F}_{1}, X\right)$.

For any $F \in \mathfrak{L} \cup\{G\}$, we call $\mathfrak{G}_{F}$ the set of Hepp's sectors of $F / \mathfrak{I}_{m} \cup \mathfrak{Q}$ which belong to $\Delta_{\mathfrak{F}_{1}, X}$. Let us recall [1] that a Hepp-sector associated to a permutation $\sigma_{F}$ is a domain in $\alpha$-space defined by:

$$
h_{\sigma_{F}} \equiv\left\{\alpha \in F ; \alpha_{\sigma(1)} \leqq \alpha_{\sigma(2)} \leqq \ldots \leqq \alpha_{\sigma\left(l_{F}\right)} \equiv \alpha_{F} \leqq 1\right\} .
$$

The Hepp variables are defined by:

$$
\alpha_{k} \equiv \prod_{k \in F_{i}^{\sigma_{F}}} \beta_{i} ; \quad 0 \leqq \beta_{i} \leqq 1,
$$

where the subgraph $F_{i}^{\sigma_{F}}$ is as in [1], the subset of $F / \mathfrak{I}_{m} \cup \mathfrak{R}$ made of the lines $\sigma(1), \ldots, \sigma(i)$, and the infra-red truncation $\alpha_{\sigma\left(l_{F}\right)}=\alpha_{F} \leqq 1$ was assumed above for simplicity.

From (B.21) and the arguments above, we obtain:

$$
\begin{aligned}
& \left|K_{G}^{\mathfrak{F}, \mathfrak{H}, \mathfrak{H}}(\mathbf{p})\right| \stackrel{n}{\leqq} \sup _{\widetilde{\mho}_{1}, X}\left[L_{\mathfrak{F}_{1}, X} \cdot \prod_{F \in \mathfrak{Q} \cup\{G\}} M_{\widetilde{F}_{1}, X}^{F}\right],
\end{aligned}
$$

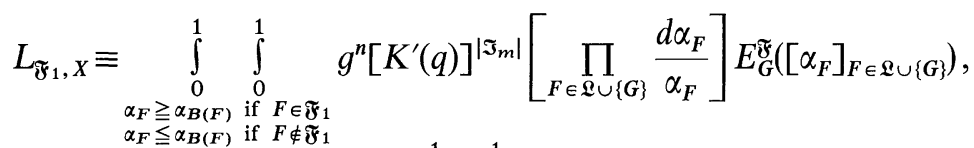

$$
\begin{aligned}
& M_{\mathfrak{F}_{1}, X}^{F} \equiv \sum_{\sigma \in \mathfrak{F}_{F}} \int_{0}^{1} \ldots \int_{0}^{1} \prod_{\substack{i \in F \\
i \neq l_{F}}} d \beta_{i} \beta_{i}^{\omega\left(F_{i}^{\sigma}\right)}-1,
\end{aligned}
$$


where $\omega\left(F_{i}^{\sigma}\right)$ is defined in Sect. (II.3) and we used $d \mu(\alpha) \leqq \prod_{i} d \alpha_{i}$. Indeed it is shown in [1], that by considering the dominant tree in each sector, the factor $\prod_{i} \beta_{i}^{\omega\left(F_{i}^{\sigma}\right)-1} d \beta_{i}$ bounds $U_{F}^{-2}\left(\alpha_{i}\right) \prod d \alpha_{i}$ in each given $h_{\sigma}$.

Let us recall that $\omega(C) \geqq \frac{1}{6} e(C)$ for any superficially convergent connected graph $C$ (Sect. II.3). Remark that if $F_{i}^{\sigma} \neq F$, hence $i \neq l_{F}, F_{i}^{\sigma}$ has no divergent connected part (otherwise they would belong to $\mathfrak{H}$, hence to $\mathfrak{I}$ by definition of $\mathfrak{H}$ and of the compatibility condition $\sigma \in \mathfrak{G}_{F}$, which means that the collection of inequalities defining $h_{\sigma}$ is compatible with the collection of inequalities defining $\left.\Delta_{\mathfrak{8}, \mathfrak{h}}\right)$. Therefore

$$
M_{\widetilde{\mho}_{1}, X}^{F} \stackrel{n_{E}}{\triangleq} \sum_{\sigma \in \mathfrak{G}_{F}} \prod_{i \neq l_{F}} \frac{1}{e_{i}^{\sigma}}
$$

Now it is proved in [1, Appendix B] that (B.29) implies $M_{\widetilde{\mho}_{1}, X}^{F} \stackrel{n_{F}}{\leqq}$. Since $\sum n_{F} \leqq 2 n$, Lemma B.1 will be the consequence of only one further lemma.

\section{Lemma B.6.}

$$
L_{\widetilde{\mho}_{1}, X} \stackrel{n}{\leqq} g^{n-\mid \mathcal{Q} \cup\{G\}}\left[K^{\prime}(q)\right]^{\left|\mathfrak{I}_{m}\right|}\left[\frac{1}{q}\right]^{|\mathfrak{I}|} .
$$

Proof. Let us make the change of variables $\gamma_{F} \equiv g \cdot c \cdot\left|\log \alpha_{F}\right|$. For $F \in \mathfrak{F}_{1}$, we have $\frac{1+\gamma_{F}}{1+\gamma_{B(F)}} \leqq 1$, hence

$$
\left(\frac{1+\gamma_{F}}{1+\gamma_{B(F)}}\right)^{2} \leqq\left(\frac{1+\gamma_{F}}{1+\gamma_{B(F)}}\right)^{3 / 2}
$$

Therefore, using (B.16):

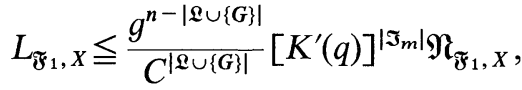

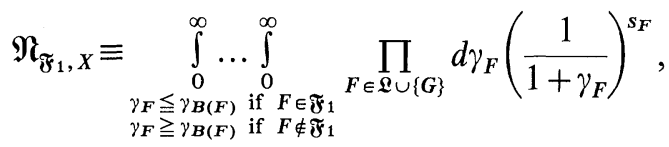

$$
\begin{aligned}
& s_{F} \equiv \#\{v \in G / B(v)=F\}+3 / 2 \#\left\{F^{\prime} \in \mathfrak{F}_{1} / B\left(F^{\prime}\right)=F\right\}-3 / 2 \chi_{\widetilde{\mho}_{1}}(F),
\end{aligned}
$$

where we introduce the characteristic function $\chi_{\widetilde{F}_{1}}$ defined by $\chi_{\widetilde{F}_{1}}(F)=1$ if $F \in \mathfrak{F}_{1}$, $\chi_{\mathfrak{F}_{1}}(F)=0$ if $F \notin \mathfrak{F}_{1} ; \chi_{\mathfrak{I}_{1}}$ and $\chi_{\mathfrak{I}}$ are defined in a similar way.

For any $F$ minimal in $\mathfrak{E} \cup\{G\}$, if $F \in \mathfrak{F}, F$ is not isomorphic to $G_{0}$ (by Definition (A.2), hence \# $\{v \in G / B(v)=F\} \geqq 3$. This implies, using definition (B.8) of $\mathfrak{I}$, that for any $F$ minimal in $\mathfrak{L} \cup\{G\}$ :

$$
s_{F} \geqq 3 / 2 \chi_{\widetilde{\mho}_{1}}(F)+3 \chi_{\mathfrak{I}_{1}}(F)+(q+1) \chi_{\mathfrak{J}}(F) .
$$


In (B.32), we integrate over $\gamma_{F_{0}}$, for $F_{0}$ minimal in $\mathfrak{L} \cup\{G\}$, using the bound (B.34). Therefore:

$$
\begin{aligned}
& \text { for } F_{0} \in \mathfrak{F}_{1}, \quad \int_{0}^{\gamma_{B}\left(F_{0}\right)} \frac{d \gamma_{F_{0}}}{\left(1+\gamma_{F_{0}}\right)^{s_{F_{0}}}} \leqq \int_{0}^{\infty} \frac{d \gamma}{(1+\gamma)^{3 / 2}} \leqq 2, \\
& \text { for } F_{0} \in \mathfrak{I}_{1}, \quad \int_{\gamma_{B\left(F_{0}\right)}}^{\infty} \frac{d \gamma_{F_{0}}}{\left(1+\gamma_{F_{0}}\right)^{S_{F_{0}}}} \leqq \int_{\gamma_{B\left(F_{0}\right)}}^{\infty} \frac{d \gamma}{(1+\gamma)^{3}} \leqq\left(\frac{1}{1+\gamma_{B\left(F_{0}\right)}}\right)^{2} \text {, } \\
& \text { for } F_{0} \in \mathfrak{I}, \quad \int_{\gamma_{B\left(F_{0}\right)}}^{\infty} \frac{d \gamma_{F_{0}}}{\left(1+\gamma_{F_{0}}\right)^{s_{F}}} \leqq \int_{\gamma_{B\left(F_{0}\right)}}^{\infty} \frac{d \gamma}{(1+\gamma)^{q+1}} \leqq \frac{1}{q}\left[\frac{1}{1+\gamma_{B\left(F_{0}\right)}}\right]^{q} \text {. }
\end{aligned}
$$

We obtain a new forest $\mathfrak{L} \cup\{G\}-\left\{F_{0}\right\}$, and a new integral similar to (B.32) with new values $s_{F}^{1} \equiv s_{F}$ if $B\left(F_{0}\right) \neq F$, and $s_{F}^{1} \equiv s_{F}+2 \chi_{\Im_{1}}\left(F_{0}\right)+q \chi_{\Im}\left(F_{0}\right)$ if $F=B\left(F_{0}\right)$. We repeat this elementary integration over a new subgraph $F_{1}$ minimal in $\mathfrak{L} \cup\{G\}$ $-\left\{F_{0}\right\}$, and so on. We have to verify that condition (B.34) never becomes violated for the new numbers $s_{F}^{1}, s_{F}^{2}, \ldots$ Let $F \in \mathfrak{L} \cup\{G\}, F=F_{k+1}$, hence $F$ becomes minimal after the $k^{\text {th }}$ integration. By induction, using (B.34) and (B.35)-(B.37) there are "reduction rules" to compute a lower bound on $s_{F}^{k}$ : any ordinary vertex in $F / \mathfrak{Q}$ counts for 1 , any $F^{\prime} \in \mathfrak{F}_{1}$ with $B\left(F^{\prime}\right)=F$ counts for $3 / 2$ [by (B.33)], any $F^{\prime} \in \mathfrak{J}_{1}$ with $B\left(F^{\prime}\right)=F$ counts for 2 [by (B.36)] and any $F^{\prime} \in \mathfrak{J}$ with $B\left(F^{\prime}\right)=F$ counts for $q$ [by (B.37)]; finally one has to subtract $3 / 2$ if $F \in \mathfrak{F}_{1}$. Using Definitions A.2 and A.3, plus (B.8), it is easy to check that this rule proves $s_{F}^{k} \geqq 3 / 2 \chi_{\widetilde{F}_{1}}(F)+3 \chi_{\mathfrak{I}_{1}}(F)+(q+1) \chi_{\mathfrak{J}}(F)$ : by induction on $\mathfrak{L} \cup\{\mathrm{G}\}$ this proves that:

$$
\mathfrak{N}_{\mathfrak{\mho}_{1}, X} \leqq 2^{n}\left[\frac{1}{q}\right]^{|\mathfrak{I}|} .
$$

[The factor 2 comes from (B.35).] Together with (B.31) this achieves the proof of Lemma B.6.

By (B.9), (B.26), (B.29), and (B.30) one gets

$$
\left|K_{G}^{\mathfrak{F}, \mathfrak{H}}(\mathbf{p})\right| \leqq C^{n} g^{n-|\mathcal{R} \cup\{G\}|}\left[K^{\prime}(q)\right]^{\left|\mathfrak{I}_{m}\right|}\left[\frac{1}{q}\right]^{|\mathfrak{s}|}
$$

for some numerical constant $C \leqq 10^{10}$. To complete the proof of (B.4), hence of Lemma (B.1), let $\eta$ be any (arbitrarily small) positive number.

We fix $q$ to be the first integer greater than $[C / \eta]^{12}$. Then we take

$$
\varepsilon<\left[K^{\prime}(q)\right]^{-1}[C / \eta]^{-12} .
$$

Remark that $|\mathfrak{F}|+|\mathfrak{I}|+|\mathfrak{I}|=|\mathfrak{F} \cup \mathfrak{H}| \leqq n$, hence $n-|\mathfrak{L} \cup\{G\}| \geqq|\mathfrak{I}|$ and $n-|\mathfrak{L} \cup\{G\}|$ $\geqq n-[|\mathfrak{F}|+|\mathfrak{H}|]$. Moreover by Lemma B.2 $|\mathfrak{F}| \leqq \frac{2 n}{3}$ and $\left|\mathfrak{I}_{m}\right| \leqq|\mathfrak{I}| \leqq|\mathfrak{H}|$. Therefore:

a) if $|\mathfrak{H}| \leqq n / 6,|\mathfrak{F}|+|\mathfrak{H}| \leqq \frac{5 n}{6}$, and:

$$
\left|K_{G}^{\mathscr{F}, \mathfrak{H}}(\mathbf{p})\right| \leqq C^{n} g^{n / 6}\left|K^{\prime}(q)\right|^{n / 6} \leqq \eta^{n}
$$


b) if $|\mathfrak{H}|>n / 6$ and $|\mathfrak{I}|<\frac{n}{12}$, hence $|\mathfrak{I}| \geqq \frac{n}{12}$ :

$$
\left|K_{G}^{\mathfrak{F}, \mathfrak{F}}(\mathbf{p})\right| \leqq C^{n}\left|K^{\prime}(q)\right|^{|\mathfrak{F}|} g^{|\mathfrak{F}|} \leqq C^{n}\left[\frac{\eta}{C}\right]^{12|\mathfrak{F}|} \leqq \eta^{n},
$$

c) if $|\mathfrak{H}|>n / 6$ and $|\mathfrak{I}| \geqq n / 12$ :

$$
\left|K_{G}^{\mathfrak{F}, \mathfrak{s}}(\mathbf{p})\right| \leqq C^{n}\left[\frac{1}{q}\right]^{n / 12}\left[g K^{\prime}(q)\right]^{|\mathfrak{F}|} \leqq C^{n}\left[\frac{1}{q}\right]^{n / 12} \leqq \eta^{n} .
$$

This completes the proof of Lemma B.1.

2. General Case. As is remarked in Sect. III, when bipeds are present it is important to do correctly the subtractions which renormalize their quadratic divergences, even in the dressed expansion. We will not use here the painful treatment of bipeds given in [1], which, as remarked in [1, Sect. IV], does not give a sharp estimate for a "chain" of bipeds. We prefer to use the following facts which allow an inductive estimate on dressed bipeds in our expansion:

a) Bipeds never overlap with closed graphs.

b) Amplitudes for bipeds $B$ depend on only one invariant, the square of the external momentum $\mathbf{p}_{B}$ flowing through them.

c) If we write the Taylor subtraction for bipeds as $1-\mathbf{t}_{B}=s_{B}^{0}+s_{B}^{1}$, where $s_{B}^{0} \equiv 1-\mathbf{t}_{B}^{0}$ subtracts only the first term in the Taylor expansion and $s_{B}^{1} \equiv \mathbf{t}_{B}^{1}$ retains the second one, each piece corresponding to $s_{B}^{0}$ or $s_{B}^{1}$ does not show any quadratic divergence, but only a logarithmic one. Moreover the operators $s_{B}^{0}$ or $s_{B}^{1}$ renormalize automatically the spurious divergences associated to open quadrupeds whose closure is $B$ [1].

d) Since by insertion of a biped the number of (ordinary) vertices of a graph increases by at least 2 there are no subtleties similar to the preceding section, and the logarithms of the dressing factor can be used to screen the remaining logarithmic divergence of the bipeds.

Let us make the following inductive assumptions on the renormalized dressed amplitude for a biped $B\left(K_{G}\right.$ is defined as $J_{G}$ in (III.8) with $K_{G}^{\mathbb{F}, \mathfrak{F}}$ instead of $\left.J_{G}^{\mathbb{F}, \mathfrak{F}}\right)$.

Lemma B.7. There exists $K(\varepsilon)$ with $\lim _{\varepsilon \rightarrow 0} K(\varepsilon)=0$, and

$$
\begin{array}{cc}
\text { a) } & \left|K_{B}^{0,1}\left(\mathbf{p}^{2}\right)\right| \leqq\left(\mathbf{p}^{2}+1\right)[K(\varepsilon)]^{n(B)} \\
\text { b) } & K_{B}^{0,1}\left((\mathbf{p}+\mathbf{k})^{2}\right)-K_{B}^{0,1}\left(\mathbf{p}^{2}\right)=\left[2 \mathbf{p} \cdot \mathbf{k}+\mathbf{k}^{2}\right] L_{B}^{0,1}(\mathbf{p} \cdot \mathbf{k}), \\
\text { c) } & \left.\frac{d}{d \chi} K_{B}^{0,1}\left((\mathbf{p}+\chi k)^{2}\right)\right|_{\chi=0} \equiv\left[2 \mathbf{p} \cdot \mathbf{k}+2 \mathbf{k}^{2}\right] M_{B}^{0,1}(\mathbf{p}), \\
\text { d) } & \left|L_{B}^{0,1}(\mathbf{p} \cdot \mathbf{k})\right| \leqq[K(\varepsilon)]^{n(B)} \\
\text { e) } & \left|M_{B}^{0,1}(\mathbf{p} \cdot \mathbf{k})\right| \leqq[K(\varepsilon)]^{n(B)}
\end{array}
$$

The induction is on the number $b(B)$ of bipeds which are strict subgraphs of $B$. For $b(B)=0$, Lemma B.7 can be proved easily by the techniques of [1] and of the preceding section, using the two dressing extra logarithms to bound the logarithmic divergences associated to $K_{B}^{0,1}$. Let $B$ be a biped with maximal sub- 
bipeds $B_{1}, \ldots, B_{i}, \ldots, B_{m}$. In the momentum representation for $B / \bigcup_{i} B_{i}$, there are loop momenta $\mathbf{q}_{1}, \ldots, \mathbf{q}_{j}, \ldots, \mathbf{q}_{L}$, and the line momenta $\mathbf{k}_{1}, \ldots, \mathbf{k}_{k}, \ldots, \mathbf{k}_{l}$ are linear combinations of the $\mathbf{q}_{j}$ 's and of $\mathbf{p}$. We call $\mathbf{p}_{i}$ the external momentum of $B_{i}$, and $\mathbf{k}_{1}^{0}, \ldots, \mathbf{k}_{k}^{0}, \ldots, \mathbf{k}_{l}^{0}, \ldots, \mathbf{p}_{i}^{0} \ldots$ the corresponding quantities at $\mathbf{p}=0$. As an analog of (B.13) we have the following mixed representation for $K_{B}^{0}$ (we forget unessential details like the possibility of forests of quadrupeds to add to the formulae, treating them as in the preceding section, and call $D_{B}$ the dressing factor for $B$ ).

$$
\begin{aligned}
K_{B}^{0}\left(\mathbf{p}^{2}\right)= & \left.g^{n(B)-\sum_{i}^{n} n\left(B_{i}\right)}\right\} \ldots \int d \mu(\alpha) D_{B} / \bigcup_{i} B_{i}(\alpha) \prod_{j=1}^{L} d^{4} \mathbf{q}_{j} \\
& \ldots\left\{\prod_{k=1}^{l} e^{-\alpha} k^{\mathbf{k}_{k}^{2}} \prod_{i=1}^{m}\left[K_{B_{i}}^{0}\left(\mathbf{p}_{i}\right)+K_{B_{i}}^{1}\left(\mathbf{p}_{i}\right)\right]\right. \\
& \left.-\prod_{k=1}^{l} e^{-\alpha_{k}\left(\mathbf{k}_{i}^{0}\right)^{2}} \prod_{i=1}^{m}\left[K_{B_{i}}^{0}\left(\mathbf{p}_{i}^{0}\right)+K_{B_{i}}^{1}\left(\mathbf{p}_{i}^{0}\right)\right]\right\} \\
= & g^{n(B)-\sum_{i} n\left(B_{i}\right)} \int \ldots \int d \mu(\alpha) D_{B} \bigcup_{i}(\alpha) \prod_{j=1}^{L} d^{4} \mathbf{q}_{j} \\
& \ldots\left\{\prod_{k=1}^{l} e^{-\alpha_{k} \mathbf{k}_{k}^{2}}-\prod_{k=1}^{l} e^{-\alpha_{k}\left(\mathbf{k}_{k}^{0}\right)^{2}}\right\} \prod_{i=1}^{m}\left[K_{B_{i}}^{0}\left(\mathbf{p}_{i}\right)+K_{B_{i}}^{1}\left(\mathbf{p}_{i}\right)\right] \\
& +\sum_{s=1}^{m} \prod_{k=1}^{l} e^{-\alpha_{k}\left(\mathbf{k}_{k}^{0}\right)^{2}} \prod_{1 \leqq i<s}\left[K_{B_{i}}^{0}\left(\mathbf{p}_{i}^{0}\right)+K_{B_{i}}^{1}\left(\mathbf{p}_{i}^{0}\right)\right] \\
& \cdot\left\{\left[K_{B_{s}}^{0}\left(\mathbf{p}_{s}\right)-K_{B_{s}}^{0}\left(\mathbf{p}_{s}^{0}\right)\right]+\left[K_{B_{s}}^{1}\left(\mathbf{p}_{1}\right)-K_{B_{s}}^{1}\left(\mathbf{p}_{s}^{0}\right)\right]\right\} \prod_{s<i \leqq m} \\
& \quad\left[K_{B_{i}}^{0}\left(\mathbf{p}_{i}\right)+K_{B_{i}}^{1}\left(\mathbf{p}_{i}\right)\right] .
\end{aligned}
$$

By (B.44), cancel the $2 \mathbf{p}_{s}^{0} \cdot \mathbf{p}_{s}$ term by symmetric integration, and bound the remaining $L_{B_{s}}^{0,1}$ by (B.46). Using three-fourth of the dressing logarithms in $D_{B /} \cup_{B_{i}}$ one can effectuate all integrations and prove (B.43) for $K_{B}^{0}$. [Remark that the factor $m+1$ which appears in the sum (B.48) is bounded by $l(B)-\sum_{i} l\left(B_{i}\right)$, hence by $2^{l(B)-\sum_{l} l\left(B_{i}\right)}$, a factor which grows inductively into an inoffensive $2^{l(B)}$. Also there remains a factor $g^{n(B) / 4}$ to ensure the decrease of $K(\varepsilon)$ as $\left.\varepsilon \rightarrow 0\right]$.

The rest of Lemma B.7 [(B.44)-(B.47)] can be verified in a similar way.

\section{Appendix C: Borel Summability}

This section is devoted to the proof of Theorem III.3. We replace first $S_{e}(\mathbf{p}, g)$ in (III.9) by its definition as the absolutely convergent sum (III.8) of the dressed amplitudes. The subtractions in (III.9) will modify and partly "undo" the dressing of the amplitudes with less than $k$ vertices, but it will not modify the dressed amplitudes with $k$ vertices or more. 
We write the left-hand side of (III.9) as:

$$
\sum_{G}\left[1-T_{g}^{k-1}\right] J_{G}(\mathbf{p}, g)
$$

where the operator $T_{g}^{q}$ retains the $q$ first terms in the Taylor development in $g$ near $g=0$.

To bound (C.1) and to prove (III.9), by linearity of $T_{g}$ and the usual exponential bounds on the number of graphs and forests it suffices to prove the following lemma:

Lemma C.1. Let $D_{\varepsilon}=\{g$ complex, $\operatorname{Re} g>0$ and $|g|<\varepsilon\}$. For $g \in D_{\varepsilon}$ :

$$
\left|\left(1-T_{g}^{k-1}\right) K_{G}^{\widetilde{F}, \mathfrak{5}}\right| \stackrel{k}{\leqq} k !|g|^{k}\left[K^{\prime}(\varepsilon)\right]^{\sup \left(0, \frac{n}{3}-k\right)}
$$

where $K^{\prime}(\varepsilon)$ is as in Lemma B.1, and $n=n(G)$.

Proof $1^{\text {st }}$ case. Let us suppose $n \leqq k+1$. We have

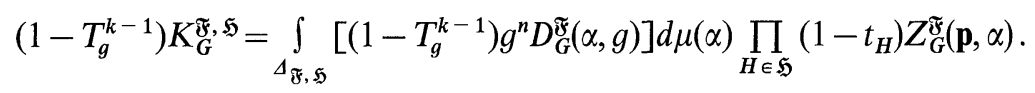

Let us call $\alpha_{\min }=\left\{\inf \alpha_{i}, i=1, \ldots, l(G)\right\}$. From the explicit form of the factors $\operatorname{od}\left(\alpha_{v}^{*}\right)$ and $\operatorname{rd}\left(\alpha_{F}, \alpha_{F}^{*}\right)$ in (III.4)-(III.5) it is obvious that $D_{G}^{\mathscr{\mho}}(\alpha, g)$ is analytic in $g$ and uniformly bounded by (const) $)^{n}$ in the set $\Gamma$ surrounding $D_{\varepsilon}$ at distance $c \cdot\left[2 \xi\left(\alpha_{\min }\right)\right]^{-1}$ (see Lemmas A.2 and A.4). By a Cauchy formula:

$$
\left(1-T_{g}^{k-1}\right)\left(g^{n} D_{G}^{\widetilde{f}}(\alpha, g)\right)=\frac{g^{k}}{2 \pi i} \oint \frac{D_{G}^{\mathfrak{\&}}(\alpha, z) d z}{z^{k-n}(z-g)} .
$$

In (C.4) the contour surrounds the segment $[0, g]$ at distance $c \cdot\left[2 \xi\left(\alpha_{\min }\right)\right]^{-1}$. Therefore one has:

$$
\left|\left(1-T_{g}^{k-1}\right)\left(g^{n} D_{G}^{\mathscr{\&}}(\alpha, g)\right)\right| \stackrel{k}{\leqq}|g|^{k}\left|\xi\left(\alpha_{\min }\right)\right|^{k+1-n} .
$$

Let us cut the integral in (C.3) into the Hepp's sectors and perform the renormalization as in [1]. It is proved in [1] that:

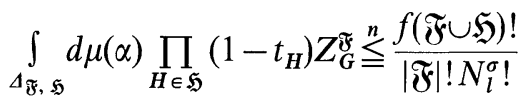

$$
\begin{aligned}
& \text {. } \sum_{\sigma / h_{\sigma} \subseteq D_{\mathscr{F}, \mathfrak{5}}} \int_{0}^{\infty} e^{-\beta_{l}} d \beta_{l} \int_{0}^{1} \ldots \int_{0}^{1} \prod_{i=1}^{1} \beta_{i}^{N^{\sigma-1}} \prod_{i=1}^{l-1} d \beta_{i},
\end{aligned}
$$

where $\beta_{i}$ are the $\beta$ variables associated to the sector $\sigma$, and $N_{i}^{\sigma}$ are positive integers and $f(\mathfrak{F} \cup \mathfrak{Y})$ and $f(G)$ are defined in [1]. Furthermore it is a basic bound of [1] that:

$$
\sum_{\sigma / h_{\sigma} \leqq D_{\mathfrak{F}, \mathfrak{F}}} \frac{f(\mathfrak{F} \cup \mathfrak{H}) !}{|\mathfrak{F}| !} \prod_{i=1}^{l-1} \frac{1}{N_{i}^{\sigma}} \stackrel{n}{\leqq} f(G) ! \leqq n ! .
$$

From Lemma A.2 and (C.5) we have in a Hepp's sector $h_{\sigma}$ :

$$
\left|\left(1-T_{g}^{k-1}\right) g^{n} D_{G}^{\widetilde{f}}(\alpha, g)\right| \stackrel{k}{\leqq}|g|^{k}\left[a+\sum_{i=1}^{l-1}\left|\log \beta_{i}\right|\right]^{k+1-n} .
$$


Using the multinomial expansion and trivial explicit integrations one gets easily:

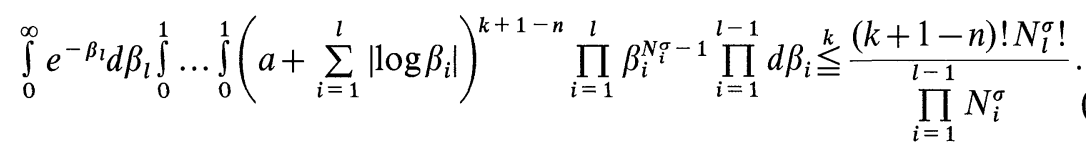

Putting together (C.7), (C.8), and (C.9) achieves the proof of Lemma C.1 since trivially $n !(k+1-n) ! \stackrel{k}{\leqq} k !$ in the case $n \leqq k+1$, and $\Delta_{\widetilde{F}, \mathfrak{H}} \subseteq D_{\widetilde{F}, \mathfrak{H}}$.

$2^{\text {nd }}$ case $k<n-1$. In this case, from the presence of $g^{n}$ in $K_{G}^{\mathbb{F}, \mathfrak{H}}$, $\left(1-T_{g}^{k-1}\right) K_{G}^{\mathbb{8}, \mathfrak{H}}=K_{G}^{\widetilde{\mho}, \mathfrak{F}}$. To prove (C.2) it remains to "trade" a factor $|g|^{k}$ against a $k$ ! in the estimates of Appendix B. Let us sketch how to do that in the case "without bipeds," namely the first part of Appendix B. The other case is similar.

We have to modify slightly the treatment of the graph $G$ in Appendix B. We distinguish two cases:

- If $|\mathfrak{H} \cup\{G\}| \geqq k$ we choose a subforest $\mathfrak{H}^{\prime}=\left\{H_{1}, \ldots, H_{l}\right\}$ of $\mathfrak{S} \cup\{G\}$ such that the $H_{j}$ 's are disjoint and such that $h \equiv \sum_{i=1}^{l} h_{i}=k$, where $h_{i} \equiv\left|\left\{H \in \mathfrak{H} \cup\{G\}, H \subseteq H_{i}\right\}\right|$.

- If $|\mathfrak{H} \cup\{G\}|<k$ we put $l=1, \mathfrak{H}^{\prime}=\{G\} \equiv\left\{H_{1}\right\}$, and $h \equiv h_{1}=|\mathfrak{H} \cup\{G\}|$.

We define also $s_{i}=\left|\left\{F \in \mathfrak{F}, F \cong H_{i}\right\}\right|, s \equiv \sum_{i=1}^{l} s_{i}$, and $f_{i}=h_{i}+s_{i}$. We define $r=\sup \{0, h+s-k-l\}$ and choose an arbitrary (possibly empty) subforest $\left\{F_{1}, \ldots, F_{r}\right\} \equiv \mathscr{F}^{\prime}$ of $\mathfrak{F}$ such that each $F_{j}, j=1, \ldots, r$ is contained in one $H_{i}$, $i=1, \ldots, l$. This is possible since $r<s$. We define also:

$$
\begin{aligned}
p_{i}^{\prime} & =\mid\left\{F \in \mathfrak{F} \cup \mathfrak{H} \cup\{G\}, F \cong H_{i} \text { and } F \cong A_{\mathscr{F}^{\prime}}\left(H_{i}\right)\right\} \mid, \\
t_{j}^{\prime} & =\mid\left\{F \in \mathfrak{F} \cup \mathfrak{S} \cup\{G\}, F \cong F_{j} \text { and } F \nsubseteq A_{\mathscr{F}^{\prime}}\left(F_{j}\right)\right\} \mid .
\end{aligned}
$$

Then one has

$$
\sum_{i=1}^{l} p_{i}^{\prime}+\sum_{j=1}^{r} t_{j}^{\prime}=\sum_{i=1}^{l} f_{i}=h+s .
$$

We define a new forest $\mathfrak{I}$ slightly different from the definition in (B.7) by:

$$
\mathfrak{I}=\left\{H \in \mathfrak{H}, n\left(H / \mathfrak{F} \cup \mathfrak{H}^{\prime}\right) \leqq q \text { and } H \nsubseteq H_{i}, i=1, \ldots, l\right\} .
$$

We write a mixed representation similar to Lemma B.3 with the corresponding new definition of $\mathfrak{I}_{m}$.

The action of the operator $T_{\widetilde{\mho}^{\prime}}$ in $K_{G}^{\mathfrak{F}, \mathfrak{G}}$ factorizes completely the various $F_{j} / \mathfrak{F}^{\prime}$, $j=1, \ldots, r$, which will be treated as in [1]. More precisely, starting from the minimal elements of 'F', one writes the bound:

$$
\begin{aligned}
& \left|\underset{\alpha^{\prime} \in \Delta(\alpha)}{\int} d \mu\left(\alpha^{\prime}\right)\left\{\prod_{v \in F_{j}}\left[\operatorname{god}\left(\alpha_{v}^{*}\right)\right]\right\} \exp \left[-2 c \zeta\left(\alpha_{F_{j}}^{*}, \alpha_{F_{j}}\right)\right] Z_{F_{j}}^{R}\left(\alpha^{\prime}\right)\right| \\
& \quad \leqq\left.(\mathrm{const})\right|_{\alpha^{\prime} \in \Delta(\alpha)} d \mu\left(\alpha^{\prime}\right) g^{n\left(F_{j}\right)-2}\left[\operatorname{god}\left(\alpha_{F_{j}}^{*}\right)\right]^{2} Z_{F_{j}}^{R}\left(\alpha^{\prime}\right) \mid
\end{aligned}
$$

where we used Lemmas A.2 and A.4. 
Performing all renormalizations as in [1] and integrating the last logarithmically divergent variable $\beta_{l}$ up to the ultraviolet cutoff $\alpha_{F_{j}}^{*}$ one gets:

$$
\begin{aligned}
& \int_{\alpha^{\prime} \in \Delta(\alpha)} d_{\mu\left(\alpha^{\prime}\right)}|g|^{n\left(F_{j}\right)-2}\left[\operatorname{god}\left(\alpha_{F_{j}}^{*}\right)\right]^{2} Z_{F_{j}}^{R}\left(\alpha^{\prime}\right) \\
& \stackrel{n\left(\underline{F}_{j}\right)}{=}|g|^{n\left(F_{j}\right)-2}\left[t_{j}^{\prime}\right] !\left|\left(\operatorname{god}\left(\alpha_{F_{j}}^{*}\right)\right)^{2} \chi\left(\alpha_{F_{j}}^{*}\right)\right| \\
& \stackrel{n\left(F_{j}\right)}{\leftrightarrows}|g|^{\left(F_{j}\right)-2}\left[t_{j}^{\prime}\right] !\left|g \operatorname{od}\left(\alpha_{F_{j}}^{*}\right)\right|,
\end{aligned}
$$

where $\chi(x)=1$ if $x>1$ and $\chi(x)=1+|\log x|$ if $x \leqq 1$. Putting the factor $\left|\operatorname{god}\left(\alpha_{F_{j}}^{*}\right)\right|$ with the rest of the integrand for $G / F_{j}$ reconstructs its proper integrand and complete dressing factors.

Iterating this argument one gets as final bound for the subgraphs of $\mathfrak{F}^{\prime}$ the factor $|g|^{n\left(\mathscr{F}^{\prime}\right)} \prod_{j=1}^{r}\left[t_{j}^{\prime}\right]$ ! [up to an inessential (const) ${ }^{n}$, where

$$
n\left(\mathfrak{F}^{\prime}\right)=\sum_{m=1}^{r^{\prime}}\left(n\left(F^{m}\right)-1\right)-\left|\mathfrak{F}^{\prime}\right|,
$$

$F^{1}, \ldots, F^{r^{\prime}}$ being the maximal elements of $\mathfrak{F}^{\prime}$, and the proper integrand and dressing factors for $G / \mathfrak{F}^{\prime}$ have been reconstructed.

The treatment of the subgraphs $H_{i} / \mathfrak{F}^{\prime}, i=1, \ldots, l$ is slightly more complicated. We consider $H_{i}^{\prime}=B_{\mathfrak{\Im}_{m}}\left(H_{i}\right)$ and define

$$
f_{i}^{\prime}=\mid\left\{F \in \mathfrak{F} \cup \mathfrak{T} \cup\{G\}, F \cong H_{i}^{\prime} \text {, and } F \nsubseteq A_{\mathfrak{F}^{\prime}}\left(H_{i}\right)\right\} \mid .
$$

By an obvious analog of Lemma B.4 applied to $H_{i}^{\prime} / \mathfrak{F}^{\prime}$ one gets:

$$
\left|\int_{\alpha^{\prime} \in \Delta(\alpha)} d \mu\left(\alpha^{\prime}\right) Z_{H_{l} / \widetilde{\mho}^{\prime}}^{R}\left(\alpha^{\prime}, \mathbf{p}_{H_{i}^{\prime}}\right)\right| \stackrel{n\left(H_{i}^{\prime} \mid \widetilde{\vartheta}^{\prime}\right)}{=} \sum_{v=1}^{f_{i}^{\prime}}\left(f_{i}^{\prime}-v\right) !\left[\alpha_{H_{i}^{*}}^{*}\left|\mathbf{p}_{H_{i}^{\prime}}\right|\right]^{v},
$$

where the natural analog of (B.14) for $H_{i}^{\prime}$ is

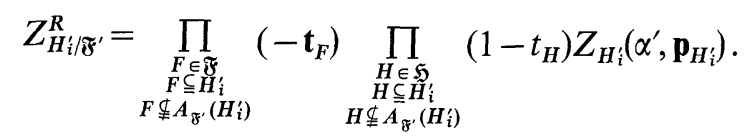

We bound $\left[\alpha_{H_{i}}^{*}\left|\mathbf{p}_{H_{i}^{i}}\right|\right]^{v}$ like in (B.19)-(B.20) by $\prod_{j=1}^{4} e^{-\alpha_{j}\left(\mathbf{p}_{H_{i}^{i}}^{j}\right)^{2}}$. We use $x^{v} e^{-x} \leqq v$ !, $v !\left(f_{i}^{\prime}-v\right) ! \leqq f_{i}^{\prime} !$ and by $($ C. 10$), f_{i}^{\prime} \leqq p_{i}^{\prime}+n\left(H_{i}^{\prime} / H_{i}\right) \leqq p_{i}^{\prime}+q$, hence $f_{i}^{\prime} ! \leqq 2^{f_{i}^{\prime}} \cdot p_{i}^{\prime} ! q !$. We remark also that

$$
\prod_{v \in H_{i}^{\prime} / \widetilde{\mathscr{V}}^{\prime}}\left|\left[\operatorname{god}\left(\alpha_{v}^{*}\right)\right]\right| \leqq|g|^{n\left(H_{i} / \widetilde{F}^{\prime}\right)-1}\left|\operatorname{god}\left(\alpha_{H_{i}}^{*}\right)\right|^{n\left(H_{i}^{\prime} / H_{i}\right)} .
$$

Putting together these bounds, the final result for integration of the subgraphs of $\mathfrak{F}^{\prime}$ and $\mathfrak{S}^{\prime}$ is a factor

$$
|g|^{\sum_{i=1}^{l}\left(n\left(H_{i}\right)-1\right)-\left|\mathscr{F}^{\prime}\right|} \prod_{j=1}^{r}\left[t_{j}^{\prime} !\right] \prod_{i=1}^{l}\left[p_{i}^{\prime}\right] !
$$

up to an inessential (const) ${ }^{n}$; the proper integrand and dressing factors for $G / \mathfrak{H}^{\prime}$ is indeed reconstructed; the factor $q$ ! is absorbed in the definition of the factor $K^{\prime}(q)$ corresponding to $H_{i}^{\prime}$ in (B.21). Hence treating $G / \mathfrak{S}^{\prime}$ as in Appendix B, we get for this 
part a bound $K(\varepsilon)^{n\left(G / \mathfrak{S}^{\prime}\right)} \cdot$ Defining $n^{\prime}=\sum_{i=1}^{l} n\left(H_{i}\right)$, gives the final bound:

$$
\left|K_{G}^{\mathbb{F}, \mathfrak{S}}\right| \leqq|g|^{n^{\prime}-l-r}[K(\varepsilon)]^{n-n^{\prime}+l} \prod_{j=1}^{r}\left[t_{j}^{\prime}\right] ! \prod_{i=1}^{l}\left[p_{i}^{\prime}\right] !
$$

By (B.5) one has $n^{\prime} \geqq h+s+l$. In the first case, where $h=k$, since $r<s$, this means that $n^{\prime}-l-r>k$. In the second case, where $\mathfrak{H}^{\prime}=\{G\}, n^{\prime}=n$, and $l=1$; either $r=0$ and $n^{\prime}-l-r=n-1>k$, or $r=h+s-k-l$, and again since $n^{\prime} \geqq h+s+l, n^{\prime}-l-r$ $\geqq k+l=k+1>k$.

Hence in every case one can extract a factor $|g|^{k}$ from the right-hand side of (C.13). Since one can choose $K(\varepsilon)$ such that $|g| \leqq K(\varepsilon)$, one obtains

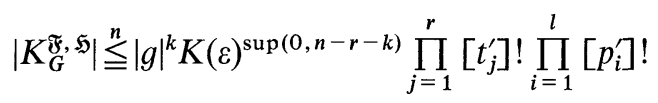

By (B.6) $r \leqq \frac{2 n}{3}$. Moreover since

$$
\sum_{j=1}^{r} t_{j}^{\prime}+\sum_{i=1}^{l} p_{i}^{\prime}=h+s, \quad \prod_{j=1}^{r}\left[t_{j}^{\prime}\right] ! \prod_{i=1}^{l}\left[p_{i}^{\prime}\right] ! \leqq \frac{(h+s) !}{(l+r) !}
$$

and:

$$
\begin{aligned}
& \text { - if } r=0, h+s<k+l \Rightarrow \frac{(h+s) !}{(l+r) !}=\frac{(h+s) !}{l !}<\frac{(k+l) !}{l !} \leqq \\
& \text { - if } r \neq 0, r=h+s-k-l \Rightarrow \frac{(h+s) !}{(l+r) !}=\frac{(h+s) !}{(h+s-k) !} \stackrel{n}{\leqq} k ! \text {. }
\end{aligned}
$$

In every case we obtain the final bound (C.2).

Acknowledgements. We thank G. 't Hooft for discussions which fostered our interest in the subject, and A. Wightman for a critical reading of our manuscript. We thank the Institute for Advanced Study for support. We have benefited from many discussions, in particular with D. Gross, E. Speer and the members of the Centre de Physique Théorique de l'Ecole Polytechnique. We are particularly grateful to E. Speer who pointed out an important mistake in an early version of this work (preprint IAS, May 1983).

\section{References}

1. de Calan, C., Rivasseau, V.: Local existence of the Borel transform in Euclidean $\Phi_{4}^{4}$. Commun. Math. Phys. 82, 69 (1981)

2. Gross, D., Neveu, A.: Dynamical symmetry breaking in asymptotically free field theories. Phys. Rev. D10, 3235 (1974)

3. Lautrup, B.: On high order estimates in QED. Phys. Lett. 69B, 109 (1977)

4. 't Hooft, G.: Lectures given at Ettore Majorana School, Erice, Sicily (1977)

5. Parisi, G.: Singularities of the Borel transform in renormalizable theories. Phys. Lett. 76B, 65 (1978)

6. Parisi, G.: The Borel transform and the renormalization group. Phys. Rep. 49, 215 (1979)

7. 't Hooft, G.: Is asymptotic freedom enough? Phys. Lett. 109B, 474 (1982)

8. 't Hooft, G.: On the convergence of planar diagram expansion. Commun. Math. Phys. 86, 449 (1982) 
9. 't Hooft, G.: Rigorous construction of planar diagram field theories in four dimensional Euclidean space. Commun. Math. Phys. 88, 1 (1983)

10. ' $t$ Hooft, G.: Borel summability of a four-dimensional field theory. Phys. Lett. 119B, 369 (1982)

11. Rivasseau, V.: Rigorous construction and Borel summability for a planar four dimensional field theory. Phys. Lett. 137 B, 98 (1984)

12. Graffi, S., Grecchi, V., Simon, B.: Borel summability: application to the anharmonic oscillator. Phys. Lett. B32, 631 (1970)

13. Eckmann, J., Magnen, J., Sénéor, R.: Decay properties and Borel summability for the Schwinger functions in $P(\phi)_{2}$ theories. Commun. Math. Phys. 39, 251 (1975)

14. Magnen, J., Sénéor, R.: Phase space cell expansion and Borel summability for the Euclidean $\varphi_{3}^{4}$ theory. Commun. Math. Phys. 56, 237 (1977)

15. Renouard, P.: Analyticité et sommabilité "de Borel" des fonctions de Schwinger du modèle de Yukawa en dimension $d=2$. I and II. Ann. Inst. H. Poincaré, 27, 237 (1977); 31, 235 (1979)

16. Eckmann, J., Epstein, H.: Time-ordered products and Schwinger functions, Commun. Math. Phys. 64, 95 (1979); Borel summability of the mass and the $S$-Matrix in $\varphi^{4}$ models 68, 245 (1979)

17. Dyson, F.J.: Divergence of perturbation theory in quantum electrodynamics. Phys. Rev. 85, 631 (1952)

18. Jaffe, A.: Divergence of perturbation theory for bosons, Commun. Math. Phys. 1, 127 (1965)

19. de Calan, C., Rivasseau, V.: The perturbation series for $\Phi_{3}^{4}$ field theory is divergent. Commun. Math. Phys. 83, 77 (1982)

20. Watson, G.: Phil. Trans. R. Soc. London Ser. A211, 279 (1912); see also Hardy, G.: Divergent Series, London: Oxford U.P. 1949

21. Nevanlinna, F.: Ann. Acad. Sci. Fenn. Ser. A12, 3 (1919)

22. Sokal, A.: An improvement of Watson's theorem on Borel summability. J. Math. Phys. 21, $261(1980)$

23. Rivasseau, V., Speer, E.: The Borel transform in Euclidean $\varphi_{v}^{4}$; Local existence for $\operatorname{Re} v<4$. Commun. Math. Phys. 72, 293 (1980)

24. Gallavotti, G., Rivasseau, V.: $\phi^{4}$ field theory in dimension 4. A modern introduction to its unsolved problems. Ann. Inst. H. Poincaré 40, 185 (1984)

25. Brydges, D., Fröhlich, J., Sokal, A.: A new proof of the existence and non-triviality of the continuum $\varphi_{2}^{4}$ and $\varphi_{3}^{4}$ quantum field theories. Commun. Math. Phys. 91, 141 (1983)

26. Aizenman, M.: Proof of the triviality of $\varphi^{4}$ field theory and some mean-field features of Ising models for $d>4$. Phys. Rev. Lett. 47, 1 (1981)

27. Aizenman, M.: Geometric analysis of $\Phi^{4}$ fields and Ising models. Parts I and II. Commun. Math. Phys. 86, 1 (1982)

28. Fröhlich, J.: On the triviality of $\lambda \varphi_{d}^{4}$ theories and the approach to the critical point in $d \geqq 4$ dimensions. Nucl. Phys. B200 (FS4), 281 (1982)

29. 't Hooft, G.: A planar diagram theory for strong interactions. Nucl. Phys. B72, 461 (1974)

30. Migdal, A.A.: Properties of the loop average in QCD, Ann. Phys. 126, 279 (1980)

31. Makeenko, Y.M., Migdal, A.A.: Quantum chromodynamics as dynamics ofloops. Nucl. Phys. B188, 269 (1981)

32. Eguchi, T., Kawai, H.: Reduction of dynamical degrees of freedom in the large $N$ gauge theory. Phys. Rev. Lett. 48, 1063 (1982)

33. Bhanot, G., Heller, U., Neuberger, H.: The quenched Eguchi-Kawai model. Phys. Lett. 113B, 47 (1982)

34. Parisi, G.: A simple expression for planar field theories. Phys. Lett. 112B, 463 (1982)

35. Gross, D., Kitazawa, Y.: A quenched momentum prescription for large $N$-theories. Nucl. Phys. B206, 440 (1982)

36. Lipatov, L.N.: Calculation of the Gell-Mann-Low function in scalar theory with strong nonlinearity. Sov. Phys. JETP 44, 1055 (1976), and Divergence of the perturbation theory series and the quasi-classical theory. JETP 45, 216 (1977)

37. Brézin, E., Le Guillou, J.C., Zinn-Justin, J.: Perturbation theory at large order. I. The $\phi^{2 N}$ interaction, and II. Role of the vacuum instability. Phys. Rev. D15, 1544, 1558 (1977) 
38. Breen, S.: PhD thesis and "Leading large order asymptotics for $\varphi_{2}^{4}$ perturbation theory." Commun. Math. Phys. 92, 197 (1983)

39. Koplik, J., Neveu, A., Nussinov, S.: Some aspects of the planar perturbation series. Nucl. Phys. B123, 109 (1977)

40. Brézin, E., Itzykson, C., Parisi, G., Zuber, J.B.: Planar diagrams. Commun. Math. Phys. 59, 35 (1978)

41. Brydges, D., Sokal, A., Spencer, T.: Private communication

42. Bergère, M., Zuber, J.B.: Renormalization of Feynman amplitudes and parametric integral representation. Commun. Math. Phys. 35, 113 (1974)

43. Bergère, M., Lam, Y.M.P.: Bogolubov-Parasiuk theorem in the $\alpha$-parametric representation. J. Math. Phys. 17, 1546 (1976)

44. Symanzik, K.: Small-distance-behaviour analysis and Wilson expansions. Commun. Math. Phys. 23, 49 (1971); Infrared singularities and small-distance-behaviour analysis. 34, 7 (1973)

45. Bogoliubov, N., Parasiuk,: Acta Math. 97, 227 (1957)

46. Hepp, K.: Proof of the Bogoliubov-Parasiuk theorem on renormalization. Commun. Math. Phys. 2, 301 (1966)

47. Zimmermann, W.: Convergence of Bogoliubov's method for renormalization in momentum space. Commun. Math. Phys. 15, 208 (1969)

48. Speer, E.: Generalized Feynman amplitudes. Princeton, NJ: Princeton University Press 1969

49. Renormalization theory. Proceedings of 1975 Erice Summer School. Velo, G., Wightman, A. (eds.)

50. de Calan, C., David, F., Rivasseau, V.: Renormalization in the complete Mellin representation of Feynman amplitudes. Commun. Math. Phys. 78, 531 (1981)

51. Landau, L., et al.: Collected papers of L. D. Landau. New York: Gordon and Breach 1965

52. Pomeranchuk, I., Sudakov, V., Ter Martirosyan, K.: Vanishing of renormalized charges in field theories with point interaction. Phys. Rev. 103, 784 (1956)

53. Redmond, P.J.: Elimination of ghosts in propagators. Phys. Rev. 112, 1404 (1958)

54. Bogoliubov, N.N., Logunov, A.A., Shirkov, D.V.: The method of dispersion relations and perturbation theory. Sov. Phys. JETP 10, 574 (1960)

55. Crutchfield, W.Y.: Phys. Rev. D19, 2370 (1979)

56. David, F.: Non-perturbative effects and infrared renormalons within the $I / N$ expansion of the $O(N)$ non-linear sigma model. Nucl. Phys. B209, 433 (1982)

Bergère, M., David, F.: In preparation

57. Wilson, K.: Non-Lagrangian models of current algebra. Phys. Rev. 179, 1499 (1969)

58. Shifman, M.A., Vainshtein, A.I., Zakharov, V.I.: QCD and resonance physics. Theoretical foundations. Nucl. Phys. B147, 385-534 (1979)

59. David, F.: Private communication

60. Glimm, J., Jaffe, A.: Positivity of the $\phi_{3}^{4}$ Hamiltonian. Fortschr. Phys. 21, 327 (1973)

61. de Calan, C., Rivasseau, V.: Comment on [1]. Commun. Math. Phys. 91, 265 (1983)

Communicated by A. Jaffe

Received May 25, 1983; in revised form July 5, 1984 